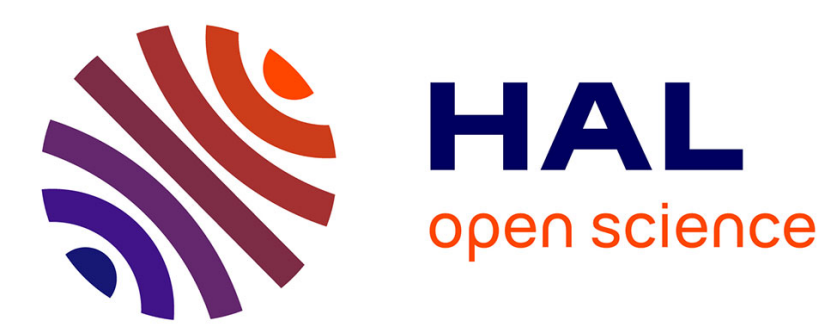

\title{
Optical Scattering Induced Noise in Fiber Ring Resonators and Optoelectronic Oscillators
}

\author{
Khaldoun Saleh, Olivier Llopis, Gilles Cibiel
}

\section{To cite this version:}

Khaldoun Saleh, Olivier Llopis, Gilles Cibiel. Optical Scattering Induced Noise in Fiber Ring Resonators and Optoelectronic Oscillators. Journal of Lightwave Technology, 2013, 31 (9), pp.1433-1446. 10.1109/JLT.2013.2250917 • hal-00800593

\section{HAL Id: hal-00800593 https://hal.science/hal-00800593}

Submitted on 14 Mar 2013

HAL is a multi-disciplinary open access archive for the deposit and dissemination of scientific research documents, whether they are published or not. The documents may come from teaching and research institutions in France or abroad, or from public or private research centers.
L'archive ouverte pluridisciplinaire HAL, est destinée au dépôt et à la diffusion de documents scientifiques de niveau recherche, publiés ou non, émanant des établissements d'enseignement et de recherche français ou étrangers, des laboratoires publics ou privés. 


\title{
Optical Scattering Induced Noise in Fiber Ring Resonators and Optoelectronic Oscillators
}

\author{
Khaldoun Saleh, Olivier Llopis, and Gilles Cibiel
}

\begin{abstract}
This paper reports both theoretical and experimental studies on nonlinear optical phenomena generated at very low thresholds in fiber ring resonators featuring ultrahigh quality factors. These studies are focused on two optical scattering phenomena: the Rayleigh and the Brillouin scatterings. The effects of these scattering phenomena on the phase noise of an optoelectronic oscillator based on the fiber ring resonator are detailed. A $30 \mathrm{~dB}$ reduction in the oscillator phase noise at $10 \mathrm{~Hz}$ offset frequency has been demonstrated by limiting the nonlinear scattering effects when using low input optical power. Moreover, a new high quality factor fiber ring resonator, optimized and immunized against some of these nonlinear optical effects, has been designed and an oscillator phase noise level of $-50 \mathrm{dBc} / \mathrm{Hz}$ at $10 \mathrm{~Hz}$ offset frequency has been achieved.
\end{abstract}

Index Terms-Fiber ring resonator, optical induced noise, optoelectronic oscillator, microwave photonics, phase noise.

\section{INTRODUCTION}

$\mathrm{H}$ IGH spectral purity microwave signals generated using classical microwave methods becomes more and more limited in the $\mathrm{X}$ to $\mathrm{V}$ bands because of the lack in frequency reference devices giving high microwave quality factors $\left(Q_{R F}\right)$ when the RF application frequency $\left(f_{R F}\right)$ increases. A wellknown classical approach is the use of multiplied quartz oscillators, which suffer from the degradation in the generated signal's phase noise by $20 \log M$ ( $M$ is the multiplication factor) [1]. Thus, multiplying a $10 \mathrm{MHz}$ signal up to $10 \mathrm{GHz}$ will degrade its phase noise by $60 \mathrm{~dB}$. Another method is the use of dielectric resonator based oscillators (DROs). These dielectric resonators feature quality factors that can reach $10^{5}$ at $10 \mathrm{GHz}$ in the case of sapphire whispering gallery modes (WGM) resonators. However, they have their own limits: their performances are degraded at high frequencies because their $Q_{R F}$ factors are inversely proportional to $f_{R F}$ and their size is prohibitive in the low frequency range $(\sim 5 \mathrm{GHz})$ [1].

Manuscript received December 10, 2012. This work was supported by the French national research agency ANR project « ORA » 2010 BLAN 0312 03, the French National Space Center (CNES) and Région Midi-Pyrénées.

K. Saleh is with the French National Space Center and LAAS-CNRS, Toulouse, 31077 France (phone: 33-5-61336879; fax: 33-5-61336969; email: ksaleh@laas.fr).

O. Llopis is with LAAS-CNRS, 31077 Toulouse, France (e-mail: llopis@laas.fr).

G. Cibiel is with the French National Space Center, 31400 Toulouse, France (e-mail: gilles.cibiel@cnes.fr).
Consequently, designing high performance oscillators is linked to the investigations on new high quality resonators.

An elegant method to reduce the oscillator dimensions and/or to increase the $Q_{R F}$ factor is to carry the microwaves on an optical wave [2]. In this case, we benefit from optical delay lines properties, such as their electromagnetic immunity, low weight and low losses. These few kilometers long delay lines can lead to high RF equivalent $Q_{R F}$ factors that can reach $10^{6}$ at $10 \mathrm{GHz}$. They have been extensively used to stabilize optoelectronic oscillators (OEOs) [3]. On the other hand, these systems face some shortcomings. They remain bulky, their thermal stabilization is difficult and they produce spurious modes that need complex configurations to be reduced [4].

An alternative solution is the use of optical resonators, featuring high optical quality factors $\left(Q_{o p t}>10^{9}\right)$ and relatively low dimensions due to the resonant effect. In this case, one solution is very similar to high $Q_{R F}$ microwave resonators: the use of optical WGM resonances. These three-dimensional shape devices present ultra-high $Q_{O p t}$ with projected values ranging from $10^{8}$ to $10^{11}$ at $1.55 \mu \mathrm{m}$ [5]. However, their use in a system is delicate because of the mechanical difficulty in setting up a reliable coupling with a light source.

Another approach is to take benefit of low loss optical fibers to make high $Q_{O p t}$ fiber ring resonators (FRRs). To predict the efficiency of the FRR in terms of quality factor and phase noise when the resonator is used as the frequency reference device in an OEO, many characterization and optimization studies have been done. One of our studies has focused on the optimization of the FRR coupling. This has led to find coupling coefficients which realize a trade-off between a high $Q_{O p t}$ and a low noise-to-carrier ratio (NCR) and has resulted in the design of relatively low phase noise FRR OEOs [6]. In addition, the FRR thermal stability has been studied and a FRR and OEO thermal stability of $6.8 \mathrm{ppm} \mathrm{K}^{-1}$ has been found [7].

In spite of these optimization studies, the OEO phase noise level was still higher than what we were theoretically predicting from an OEO model including the resonator parameters $\left(Q_{O p t}\right.$, losses, etc.) and the conversion of the main noise sources in the system. In fact, there are different optical and RF noise conversion phenomena inside the resonator itself that have to be taken into account. Among these phenomena, we can especially distinguish the laser frequency (FM) noise conversions to RF amplitude (AM) and phase (PM) noise through the resonator frequency response, particularly if the optical carrier is not perfectly centered onto one of the optical 
resonances. Furthermore, the laser AM noise conversion into RF AM and PM noise via the photodiode nonlinearities has to be avoided [8]. However, the most critical phenomena that have to be completely suppressed, in order to further reduce the oscillator phase noise, are the nonlinear optical effects generated inside the resonator due to its very high $Q_{o p t}$. The high $Q_{O p t}$ of the resonator decreases the thresholds of many power-dependent nonlinear optical effects, especially the stimulated Rayleigh scattering (STRS) and the stimulated Brillouin scattering (SBS). Of course, these two nonlinear optical phenomena are of interest for many applications [9] [12]. Moreover, it has been demonstrated in [13] that the SBS can be very useful to create an all-optical tunable OEO. However, the STRS and SBS are considered as parasitic signals in our OEO setup and they can severely degrade its phase noise.

In this paper, we first describe the principles of a high $Q_{O p t}$ FRR and of an OEO based on this device. We then present theoretical and experimental characterization studies made on the different optical scattering mechanisms generated inside the FRR, especially the Brillouin scattering and the Rayleigh scattering, and their consequences on the OEO phase noise.

\section{FIBER RING RESONATOR}

The optical fiber ring resonator, shown in Fig. 1, is fabricated using two low loss fibered $2 \times 2$ optical directional couplers $\left(\mathrm{C}_{1}\right.$ and $\left.\mathrm{C}_{2}\right)$ linked with single-mode polarizationmaintaining $(\mathrm{PM})$ fibers.

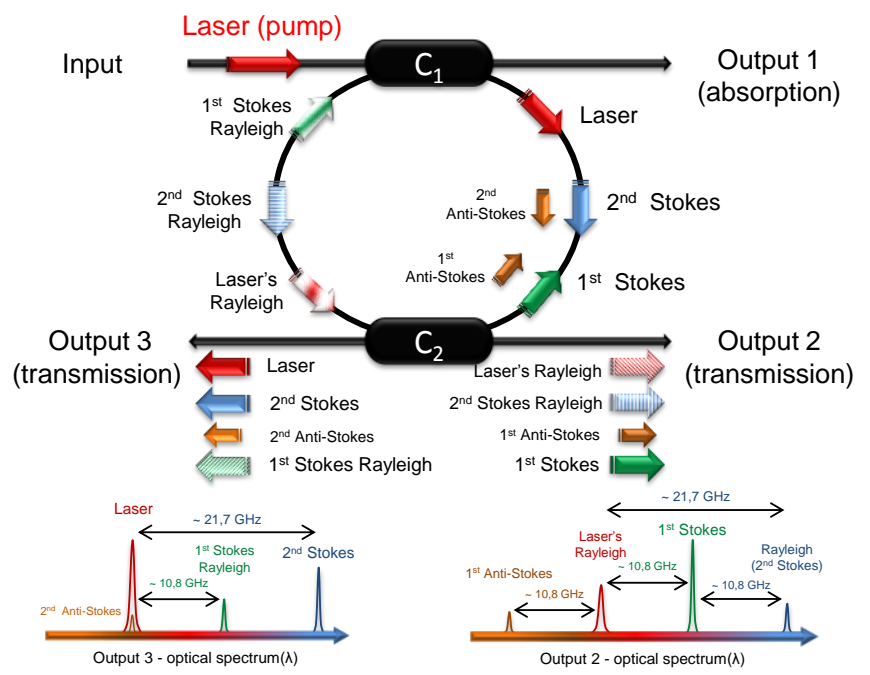

Fig. 1. Double directional coupler fiber ring resonator and the generation of the nonlinear optical effects inside with their travelling directions and their spectral representations in the optical domain. Stokes and anti-Stokes terms refer to SBS waves only.

Resonance occurs if the total integrated phase shift of the incident laser wave $\left(\lambda_{l} \sim 1.55 \mu \mathrm{m}\right)$ around the resonator ring is an integer multiple of $2 \pi$ radian. The resonator will thus generate a transverse single frequency comb with microwave spacing called free spectral range, $(F S R=c / n L)$, directly linked to the fiber length $(L)$, its refractive index $(n)$ and to the light speed in free space $\left(c \sim 3.10^{8} \mathrm{~m} / \mathrm{s}\right)$.

The quality factor of the resonator depends on the couplers' coupling coefficients $\left(\kappa_{1}\right.$ for $\mathrm{C}_{1}$ and $\kappa_{2}$ for $\left.\mathrm{C}_{2}\right)$ and the overall losses in the resonator architecture (couplers excess loss $\gamma_{1,2}$, splices loss $\alpha_{s}$ and fiber linear loss $\alpha_{f}$ ). One of the advantages of this resonator is that the $3 \mathrm{~dB}$ bandwidth of its optical resonance is maintained when used for microwave filtering in the microwave domain [14]. Therefore, the equivalent $Q_{R F}$ factor is linked to the $Q_{O p t}$ factor and it is directly proportional to the microwave application frequency $f_{R F}$. This relation is given as follows [14]:

$$
Q_{R F}=Q_{O p t} \cdot \frac{f_{R F}}{f_{O p t}}
$$

where $f_{\text {Opt }}$ is the laser carrier frequency $(\sim 193 \mathrm{THz})$.

From this relation, it is clear that the resonator $Q_{R F}$ increases with the RF application frequency for a fixed optical frequency, which is not the case in microwave resonators. Thus, to reach equivalent RF quality factors higher than those obtained in microwave resonators, $Q_{o p t}$ should be higher than $10^{9}$ because of the frequency ratio between $f_{R F}$ and $f_{O p t}$. This ratio is about $10^{-4}$ if a $1.55 \mu \mathrm{m}$ laser carrier $(\sim 193 \mathrm{THz})$ and a microwave signal around $20 \mathrm{GHz}$ are used.

Generally, if the coupling coefficients are chosen small enough, and if the residual losses of all elements in the resonator are low enough, the constructive interference between $\lambda_{l}$ and its replica inside the resonator makes the FRR circulating intra-cavity power $\left(P_{\text {Cavity }}\right)$ grow until the power dissipated in the resonator loop equals the input power $\left(P_{I n}\right)$. This optical resonator will thus feature a high $Q_{O p t}$ factor and photons inside the resonator will encounter an optical delay $\tau$ as if they are travelling into an equivalent optical fiber length, in single-pass-geometry (SPG), [14]. This equivalent length $\left(L_{e q}\right)$ is given by [14]:

$$
L_{e q}=\frac{Q_{O p t} \cdot c}{n \cdot \pi \cdot f_{O p t}}
$$

Consequently, a resonator with a $Q_{o p t}$ of $3.5 \times 10^{9}$ ( $Q_{o p t}$ factor achieved in a 20m-long FRR and being accurately measured using the method described in [15]) is equivalent to a $1180 \mathrm{~m}$-long optical delay line. This high $Q_{O p t}$ leads to a significant circulating power [16], $\left(P_{\text {Cavity }} / P_{I n} \sim 54\right.$; value simulated for a $20 \mathrm{~m}$-long FRR and experimentally estimated via the FRR output coupler's coupling coefficient), which in turn reduces the thresholds of many nonlinear optical effects.

\section{OPTOELECTRONIC OSCILLATOR}

Our $10 \mathrm{GHz}$ OEO based on a FRR, is presented in Fig. 2. It includes a narrow linewidth Koheras laser $(\sim 1 \mathrm{kHz})$, a MachZehnder modulator (MZM), a FRR, an erbium doped fiber 
amplifier (EDFA) and a fast photodiode. The laser frequency is then stabilized onto one of the ring resonances using a low frequency Pound-Drever-Hall (PDH) feedback loop, well explained in [17]. The electro-optic modulator (EOM) in the OEO setup is a part of the PDH laser stabilization loop and it is used to phase modulate the laser carrier (the PDH modulation frequency is of a few $\mathrm{MHz}$ ). The microwave oscillation is then maintained by a high frequency loop including two RF amplifiers, a phase shifter and a RF filter in order to get a single mode oscillation. This OEO is capable of generating high spectral purity signals that can be locally used from its RF output, or directly distributed via long distance and low loss optical links, using the optical output.

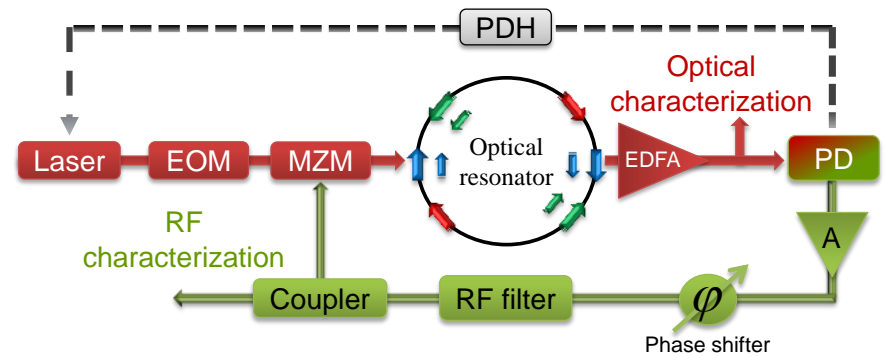

Fig. 2. $10 \mathrm{GHz}$ optoelectronic oscillator based on a fiber ring resonator. The $\mathrm{EOM}$ is used in the PDH loop to phase modulate the laser carrier.

In spite of various attempts to optimize the phase noise of this OEO, the phase noise level remained relatively high (see the red curve in Fig. 12). We have found that nonlinear optical scattering mechanisms, generated inside the high $Q_{O p t}$ FRR, were the main reason behind most of the $1 / f$ noise observed in this OEO. This behavior was directly linked to the very high $Q_{O p t}$ value of $3.5 \times 10^{9}$. Indeed, the OEO phase noise was very dependent on the onset of the nonlinear optical effects inside the resonator, which depends on the input optical power injected at the resonator input. Working at optical power levels below the threshold of the dominant nonlinear optical effect, the Brillouin scattering, has led to a considerable reduction of the OEO's close-to-carrier phase noise. However, even with a low optical power $(<-9 \mathrm{dBm})$, the OEO's close-to-carrier phase noise was far from having a classical $1 / f^{3}$ noise shape. Furthermore, operating at low optical power levels degrades the NCR of the optical link. This has led us to deeply investigate the nonlinear optical effects generated inside the 20m-long FRR, especially the SBS and the STRS, to find the solution that leads to an optimized FRR with a very high $Q_{O p t}$ and being immunized against these nonlinear optical phenomena at the same time.

\section{NONLINEAR OPTICAL MECHANISMS}

In optical fibers, many optical scattering phenomena may occur, especially at high optical power levels [18]. Among these, the well-known phenomena are Rayleigh, Brillouin and Raman scatterings. These active nonlinear phenomena feature two scattering modes each: spontaneous and stimulated scatterings, and they generally depend on molecular or density variations of silica in optical fibers. Also, another nonlinear optical effect could be generated in optical fibers and has to be mentioned: the four-wave mixing (FWM).

In the OEO case, scattering mechanisms induce noise in the spectra of optical signals travelling through the optical fiber. This fiber-induced noise degrades the low-noise signals transmitted via optical fibers [19]. In the following sections, we give a description of each of these phenomena and their possible contribution to the noise induced in our OEO setup.

\section{A. Fresnel Back-reflection}

The Fresnel back-reflection phenomenon causes light to counter-propagate in an optical fiber, and is often due to the index change from the glass-air interfaces at the fiber connections. It can be avoided by using optical fibers with cleaved connectors (cleaving angle $\sim 8^{\circ}$ ) [20], or by using index matching gel between the fibers' connectors. In our OEO setup, we use angled-polished connectors (FC/APC), so we assume that this type of reflection is considerably minimized (there are no connectors inside the FRR).

\section{B. Stimulated Brillouin Scattering}

\section{1) Stimulated Brillouin Scattering in Optical Fiber and FRR:}

Theoretically predicted by L. Brillouin in 1922 [21], the SBS in optical fibers was firstly observed in 1964 and has been extensively studied since then [22]. It is recognized as the dominant optical fiber nonlinearity and is caused by the interaction between a light wave and an acoustic wave existing already at the optical medium's thermal equilibrium. This acoustic wave travels at sound speed in the optical fiber $\left(c_{a c} \sim\right.$ $6 \mathrm{~km} / \mathrm{s}$ ) and produces a periodic modulation of its refractive index. This acts like a moving fiber Bragg grating that refracts a part of the incident light wave. The interaction creates a scattered wave; in this case we are in the spontaneous Brillouin scattering regime where the optical material properties are not affected by the presence of the incident light wave.

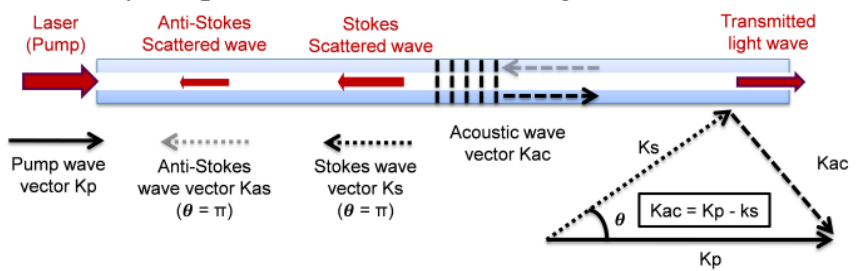

Fig. 3. Wave vector diagram illustrating SBS efficiency in the backward direction in optical fibers.

In optical fibers, Fig. 3, the acoustic wave frequency $\left(v_{a c}\right)$ depends on the speed of sound in the fiber $c_{a c}$, the fiber effective refractive index $n$, the laser carrier wavelength and the scattering angle $(\theta)$ between the wave vectors of the carrier and the scattered wave. It is given by the relation below:

$$
v_{a c}=2 c_{a c} \cdot \frac{n}{\lambda_{l}} \cdot \sin \left(\frac{\theta}{2}\right)
$$


We can see from (3) that in an optical fiber, the backscattered Stokes wave is dominant and is frequency downshifted from that of the incident light wave by an amount $v_{\mathrm{B}}$ set by the nonlinear medium characteristics $\left(v_{B} \sim v_{a c}\right)$. This frequency shift is about $11 \mathrm{GHz}$ in silica fibers [18]. This negative frequency shift (Stokes wave) is caused by the Doppler effect of the interaction between the light wave and the acoustic wave part (moving Bragg mirror) travelling in the same direction of the light. However, a very weak scattered wave, travelling in the same direction of the Stokes wave and called anti-Stokes, but up-shifted in frequency by $v_{\mathrm{B}}$, can be also generated. Its positive frequency shift is due to the interaction of the light wave with a counter-propagating acoustic wave.

At a critical incident optical power, called SBS threshold (SBST), the nature of the scattering process grossly modifies the optical properties of the medium, and the interference between the Stokes wave and the incident light wave amplifies the acoustic wave in a process called electrostriction (that is the tendency of materials to become denser in regions of high optical intensity). Thus, the scattered wave will become more intense and reinforces the interference and so on; we are now in the stimulated regime.

The optical carrier depletion occurs at power levels above the SBST because the additional optical power injected in the fiber will be converted into the Stokes wave via the electrostriction process (the anti-Stokes wave contribution to the scattering process is very weak). When the Stokes power exceeds the medium SBST, it creates a second order Stokes wave downshifted in frequency from the first Stokes wave by $v_{B}$, and thus by $2 v_{B}$ from the incident light wave.

The theoretical spectral representation in the optical domain of the first and second order SBS waves (Stokes and antiStokes waves) is depicted in Fig. 4. The representation also includes the SBS's own Rayleigh-scattered waves with their small frequency shift (few $\mathrm{kHz}$ ). The Rayleigh scattering is discussed in the next section. The travelling directions of these scattered waves in the FRR are already represented in Fig. 1.

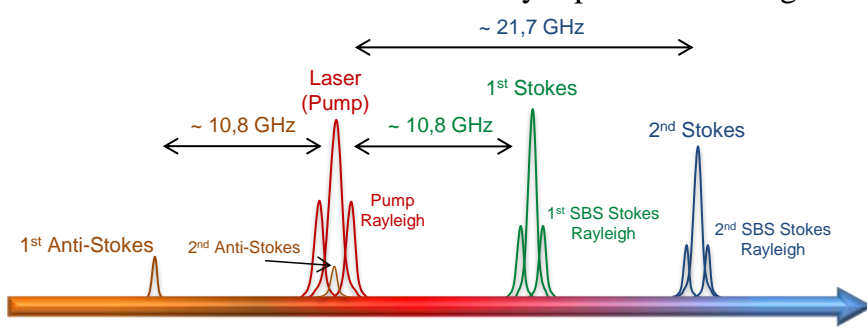

Fig. 4. Theoretical optical spectrum representation of the first and second order SBS waves and their own Rayleigh-scattered waves at the resonator's combined second and third outputs. Stokes and anti-Stokes terms refer to SBS waves only.

The SBST in SPG optical fiber is drastically reduced if the same fiber length is used in FRR geometry. The SBST reduction factor is directly linked to the resonator $Q_{o p t}$. Different SBST definitions can be found in the literature. It is generally defined as the power input at which the scattered power measured at the fiber input becomes equal to the transmitted power measured at the fiber output. In [18], the definition of the SBST is given as:

$$
P_{t h-S B S-S P G}=21 \cdot \frac{A_{e f f} \cdot K}{g_{0} \cdot L_{e f f}}
$$

where $A_{\text {eff }}\left(\mathrm{m}^{2}\right)$ is the fiber effective core area: $A_{\text {eff }}=\pi \omega^{2}$ $\left(\omega\left(\mathrm{m}^{2}\right)\right.$ being the mode radius or light spot radius), $g_{0}$ is the maximum Brillouin gain value for silica fibers $\left(g_{0} \sim 4.10^{-11} \mathrm{~m} / \mathrm{W}\right), K$ is the polarization factor $\{K=1$ in PM fibers, where the laser carrier and Stokes wave interaction is maximum due to the polarization state match between the two travelling waves, and $K=1.5$ in single mode SMF fibers $\}$ and $L_{\text {eff }}$ is the fiber effective length.

A longer effective length provides more efficient nonlinear interactions in the fiber, which may hamper a normal transmission of the optical signal. $L_{\text {eff }}$ is given by:

$$
L_{e f f}=\frac{1-e^{-\alpha_{f} \cdot L}}{\alpha_{f}}
$$

Using (4) and (5), with $L=20 \mathrm{~m}$ (the fiber real length), $\alpha_{f}=1.1510^{-4} \mathrm{~m}^{-1}$ and $A_{\text {eff }}=86 \mu \mathrm{m}^{2}$ we get a SBST $P_{t h-S B S-S P G}=2.27 \mathrm{~W}(33.5 \mathrm{dBm})$.

Now if we consider the FRR equivalent length defined in (2), we can extend equation (4) to get an estimation of the SBS intra-cavity power threshold $\left(P_{t h-S B S-I C}\right)$ in a FRR (assuming that the Brillouin frequency shift $v_{\mathrm{B}}$ is an integer multiple of the FRR's FSR and its gain spectrum is therefore centered on one of the FRR's resonances). Therefore, $L_{\text {eff }}$ in (4) will be replaced by the resonator equivalent length given in function of its quality factor in (2). Using this approach, in a 20m-long FRR with $Q_{O p t}=3.5 \times 10^{9}, P_{t h-S B S-I C}$ will be equal to $38 \mathrm{~mW}$ $(15.8 \mathrm{dBm})$, instead of $2.27 \mathrm{~W}(33.5 \mathrm{dBm})$ for the same fiber length in SPG.

However, this approach gives only the FRR's intra-cavity SBST. In order to get the FRR's input power SBST, $\left(P_{t h-S B S-R}\right)$, we have to use the relation given in [9], which gives an approximation on the power enhancement factor between the input power and the circulating power inside the resonator:

$$
\frac{P_{t h-S B S-I C}}{P_{t h-S B S-R}}=\frac{1-\gamma_{1}}{1-\left(1-\kappa_{1}\right)}
$$

In our 20m-long FRR, the $\mathrm{C}_{1}$ coupler's fractional power loss $\gamma_{1}$ is close to $0.7 \%(0.03 \mathrm{~dB})$ and its coupling coefficient $\kappa_{1} \sim 1.9 \%$. $P_{\text {th-SBS-R }}$ will be then 52 times $(17.1 \mathrm{~dB})$ lower than $P_{\text {th-IC }}$, (a power enhancement factor of $\sim 54$ has been also simulated and experimentally estimated) with a value of $0.73 \mathrm{~mW}(-1.4 \mathrm{dBm})$. If we compare $P_{t h-S B S-R}$ and $P_{t h-S B S-S P G}$, we will find a SBST reduction factor of $34.9 \mathrm{~dB}$ between a $20 \mathrm{~m}$-long fiber in SPG and the same fiber length in the FRR 
geometry (of course, the FRR must be optimized in order to get a very high $Q_{o p t}$, thus a very long $L_{e q}$, and a high power enhancement factor). Such a significant SBST reduction in a FRR will have detrimental effects on our OEO at $10 \mathrm{GHz}$ due to the presence of high order Brillouin lines in the optical spectrum, and therefore in the RF spectrum of the OEO $(\sim 11 \mathrm{GHz}, 22 \mathrm{GHz} \ldots)$, see Fig. 4.

2) SBS Frequency Shift and Gain Spectrum Characterization:

In order to investigate the SBS generated inside the FRR, we had first to determine its frequency shift and gain spectrum. In fact, many Brillouin gain spectrum (BGS) full width at half maximum (FWHM) values have been reported in the literature for silica fibers: $\Delta v_{B}=20 \mathrm{MHz}$ in [22] for SMF28 fibers and $33 \mathrm{MHz}$ in [23] for PM panda fibers, but less than $10 \mathrm{MHz}$ in [10] also for a PM fiber. Due to these different reported values, we have chosen an accurate method, well described and explained in [24], to measure the BGS in our 20m-long resonator under test (RUT).

Of course, in [24] the method is used to characterize the SBS in SPG fibers. However, the FRR frequencies are discrete, so the resolution of the BGS characterization will depend on the resonator FSR. In the 20m-long FRR, the FSR is around $10 \mathrm{MHz}$, so the use of this method will give us a measurement point at each $10 \mathrm{MHz}$ frequency step, which is enough to predict the FWHM of the Brillouin gain. The experimental measurement bench is presented in Fig. 5.

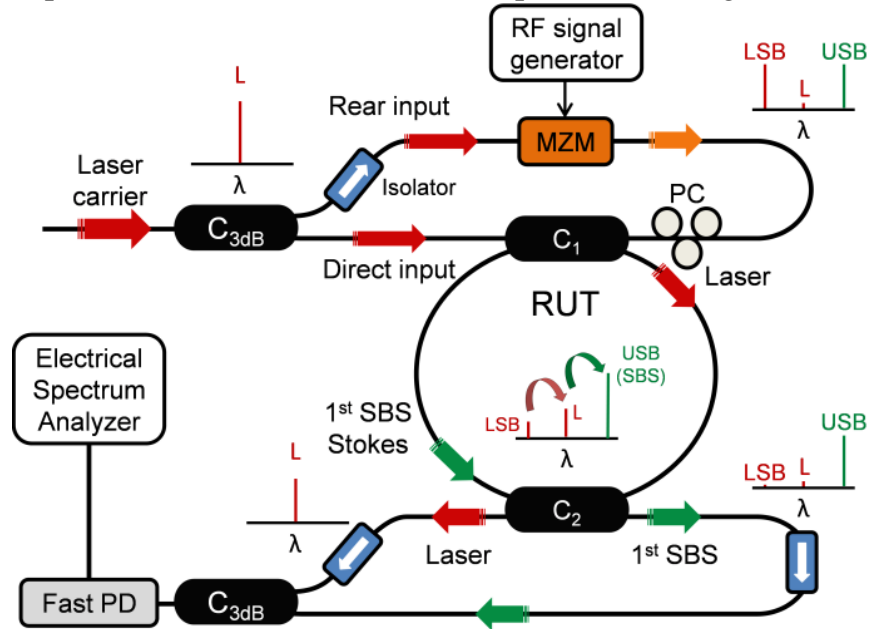

Fig. 5. Experimental bench used to characterization the Brillouin gain spectrum in a FRR. PC: polarization controller; $\mathrm{C}_{3 \mathrm{~dB}}$ : $3 \mathrm{~dB}$ coupler; RUT: resonator under test. Stokes and anti-Stokes terms refer to SBS waves only.

The only difference with [24] is that the SPG optical fiber is replaced by the FRR geometry, and that we use a very low carrier power at the resonator direct input because of the FRR intra-cavity power enhancement factor $(\sim 54)$. This is done in order to only generate the spontaneous Brillouin scattering at the beginning and not the SBS. Also, the laser carrier is stabilized onto the resonator using the PDH loop, and the carrier is phase modulated at $10 \mathrm{MHz}$ frequency via the EOM. Interestingly, it seems that this phase modulation has no suppressing effect on either the SBS or the Rayleigh scattering in our FRR, contrarily to what it is usually observed in SPG fibers [19].

The MZM DC bias voltage is set in order to get a double sideband suppressed carrier modulation; this prevents the interference between the laser carrier's two parts circulating inside the resonator in opposite directions. The BGS is then measured by sweeping the frequency of the RF driving signal of the MZM and recording the photodiode response at the FRR's combined second and third outputs, using an electrical spectrum analyzer (ESA). A low RF power of the driving signal is used to get a low power in the resulting modulation optical sidebands and to be sure that the resulting RF signal visualized at the ESA is the result of the BGS amplification.

When the wavelength of the modulation upper sideband (USB) falls within the BGS, it takes benefit of its gain and it is thus amplified by the process called Brillouin selective sideband amplification (BSSA) [25]. In this architecture, many optical interactions occur as follows: the direct propagating laser carrier (DPC) generates the spontaneous BGS inside the resonator, then the modulation USB interacts with the DPC and grows exponentially via the BSSA and the electrostriction processes, depending on the modulation USB frequency regarding the BGS frequency shift. In addition, the modulation lower sideband (LSB) can feed the DPC by the same process, but this case is insignificant because the LSB power is very low compared to the DPC power. Finally, the beat note of the DPC and the SBS waves from the resonator's combined outputs on a fast photodiode provides an accurate analysis of the BGS using the ESA.

During the BGS characterization, we have found the same dependence of the BGS on the polarization of the pump as reported in [24]. We have therefore set the MZM output's polarization in order to get the maximum BGS. The results of the BGS characterization in a $20 \mathrm{~m}$-long FRR are depicted in Fig. 6.

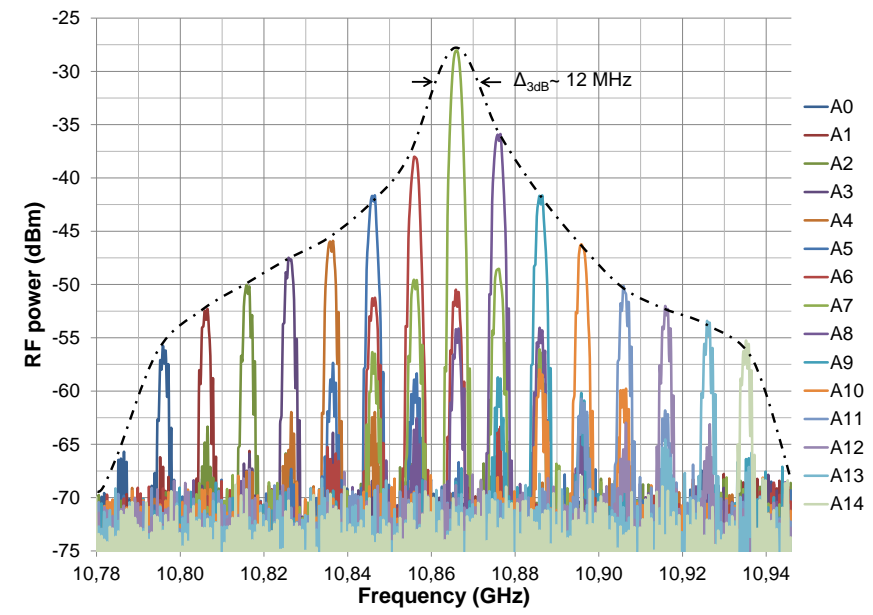

Fig. 6. 20m-long FRR Brillouin gain spectrum characterized using the experimental setup of Fig. 5. We sweep the RF modulation frequency and we take an acquisition on the ESA at each resonance of the FRR. We manage the polarization of the MZM output in order to get the maximum BGS.

The results give a Brillouin frequency shift of $10.868 \mathrm{GHz}$ and a gain FWHM of about $12 \mathrm{MHz}$. As a consequence, we are sure to have a quasi-single mode Stokes 
wave operation inside the FRR with a good rejection of the Brillouin gain filtered sidebands (at least $20 \mathrm{~dB}$ rejection of the first sidebands; see the A7 acquisition in Fig. 6).

\section{3) SBS Power Characterization:}

Before performing a power characterization of the SBS, we have first directly measured the power of the transmitted laser carrier at the FRR third output when injecting a very low laser power at the FRR input (less than $-12 \mathrm{dBm}$ ). In this case, we can assume that there is almost no additional nonlinear loss inside the FRR. The FRR loss between the input port and the third output port has been found to be equal to $3.7 \mathrm{~dB}$.

After that, the power of the different SBS Stokes waves generated inside the resonator have been measured as a function of the laser input power in the optical domain, using the experimental setup depicted in Fig. 7. Moreover, this setup has been used to characterize both optical and RF spectra at the resonator's combined outputs.

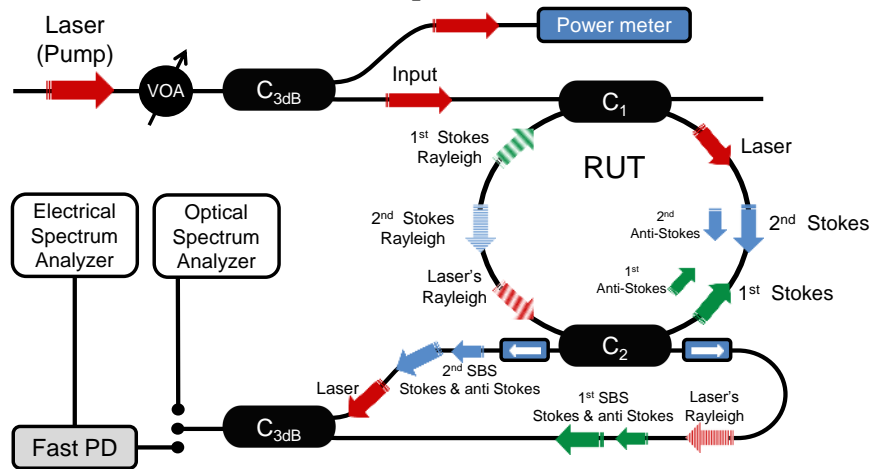

Fig. 7. Experimental setup for nonlinear optical effects characterization in the FRR. VOA: variable optical attenuator; PD: photodiode; $\mathrm{C}_{3 \mathrm{~dB}}$ : $3 \mathrm{~dB}$ coupler. Stokes and anti-Stokes terms refer to SBS waves only.

In this setup, the laser (pump) beam is split equally by means of a $3 \mathrm{~dB}$ optical coupler. The first part is sent to the resonator input and the second part is sent to an optical power meter in order to measure the optical input power level. The different measurements are then performed at the resonator outputs. The optical measurements and spectrum characterization were performed using an Agilent HP 86142A optical spectrum analyzer (OSA) with $0.06 \mathrm{~nm}$ resolution and $\pm 1 \mathrm{~dB}$ amplitude precision. The RF spectrum characterization was performed using a Rohde \& Schwarz $67 \mathrm{GHz}$ ESA.

Fig. 8 shows the measured power levels of the first four SBS Stokes waves, the laser carrier and the laser's Rayleighscattered wave. Certainly, it will be impossible to distinguish each spectrum of these different optical components if we rely only on the resolution of the OSA. But the resonator geometry helps to easily distinguish between them because of their different travelling directions (see Fig. 1).

We can clearly see that the experimental first SBST is around $-3.5 \mathrm{dBm}$ according to the definition in [18] (the input power at which the scattered power becomes equal to the transmitted power). This seems to fit with our theoretical approximation on the FRR SBST using (2), (4) and (6), with a $2 \mathrm{~dB}$ error. This error could be due first to the OSA power accuracy, and then to the SBST definition itself [18]. Indeed, even with $5 \mathrm{~dB}$ under the $-3.5 \mathrm{dBm}$ input power value, we notice that the first Stokes wave is already in a stimulated regime and that the laser output starts to saturate at $-8 \mathrm{dBm}$ input power because of the SBS, which starts to convert the laser's additional input power via electrostriction. This carrier depletion causes the NCR degradation of the optical link.

The first Stokes wave also starts to saturate at $-3 \mathrm{dBm}$ input power when the second Stokes wave starts to be in a stimulated regime and converts the first Stokes wave's power. The little increment in the laser output power between $-3 \mathrm{dBm}$ and $-1 \mathrm{dBm}$ input power levels is due to the second SBS antiStokes wave that overlaps with the laser carrier wave and increases its power (see Fig. 4).

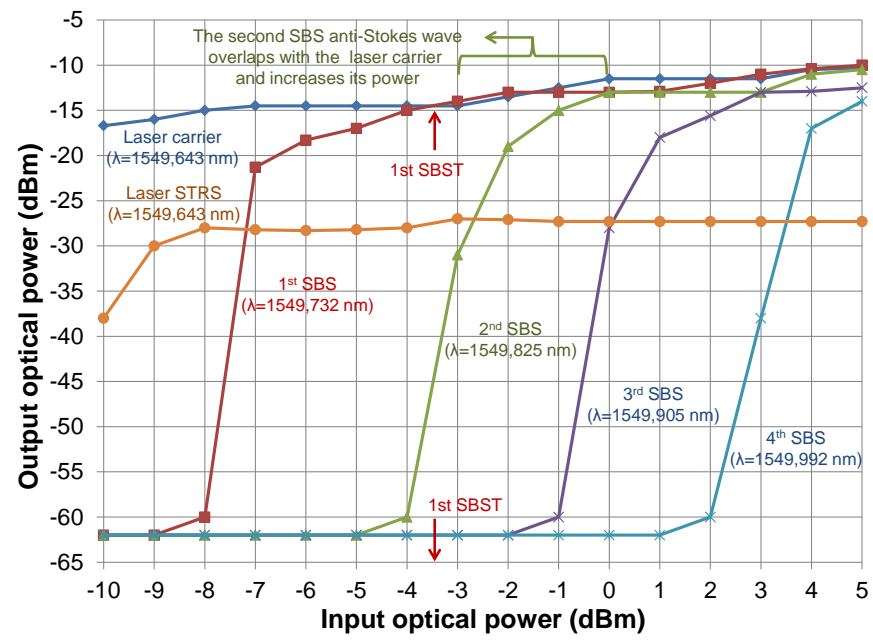

Fig. 8. Experimental power measurements results of the laser output, laser's Rayleigh-scattered wave and SBS waves, in a 20m-long FRR, versus the laser input optical power.

The optical spectrum of the resonator's combined outputs has been also measured on the OSA for different laser input power levels using the experimental setup of Fig. 7. Optical spectrum results are presented in Fig. 9. The odd order SBS waves are emitted from the resonator's second output and the even order SBS waves are emitted from the resonator's third output, as illustrated in Fig. 1. These different SBS orders were spaced by $0.088 \mathrm{~nm}(10.86 \mathrm{GHz})$ from each other and interestingly, the seventh SBS order can be generated with less than $10 \mathrm{~mW}(10 \mathrm{dBm})$ of input power. It has also less than $8 \mathrm{~dB}$ power gap with the laser output power and it is spaced from it by $\sim 0.616 \mathrm{~nm}(76 \mathrm{GHz})$.

The beat note of this optical spectrum, while injecting a $10 \mathrm{dBm}$ input power, has also been measured using a fast photodiode ( $\mathrm{U}^{2} \mathrm{t}$ photodiode with $75 \mathrm{GHz}$ bandwidth) on the $67 \mathrm{GHz}$ ESA. The results are presented in Fig. 10. The stability of the obtained RF spectrum and each of the Stokes waves depends on the resonator thermal stability and especially on the laser stabilization onto the resonance center by means of the PDH loop. The use of such nonlinear optical effect to generate millimeter wave signals around $76 \mathrm{GHz}$ could be a very promising technique. Moreover, it is also interesting to point out that the spectral linewidth of these 
Stokes waves seems to be of few $\mathrm{kHz}$ (a FWHM of approximately $25 \mathrm{kHz}$ has been measured for the first SBS Stokes wave). This is due to the resonator's narrow bandwidth filtering effect, as a result of its very high $Q_{O p t}$.

In addition, it has been reported in [26] - [29] that the laser phase noise is transferred to the emitted SBS Stoke wave after being strongly reduced and smoothed, and that the linewidth of the Stokes wave becomes narrower than that of the pump laser. Therefore, we have used the BSSA process in the 20m-long FRR to generate high power and high spectral purity millimeter wave signals. This study is well detailed in [12].

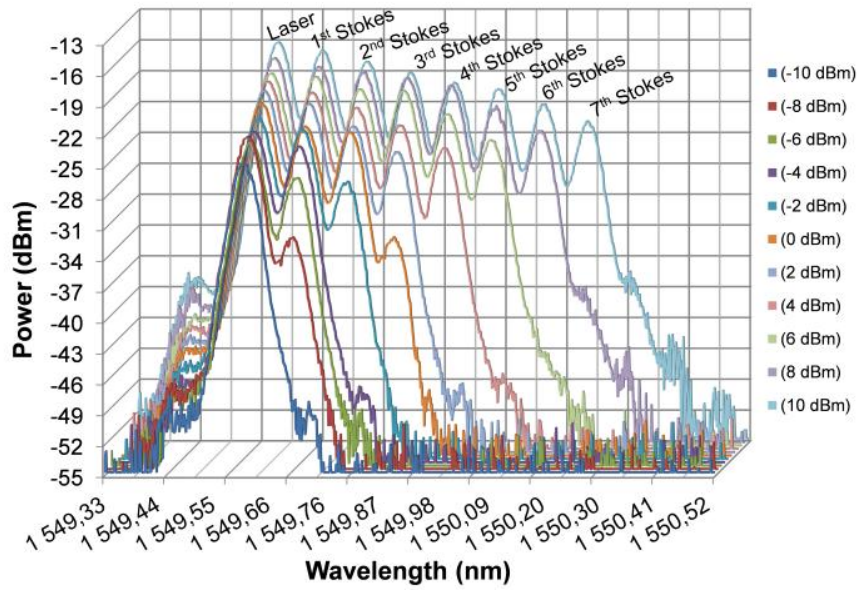

Fig. 9. Multiple acquisitions for the optical spectrum at the 20m-long FRR's combined outputs, for different laser input power levels.

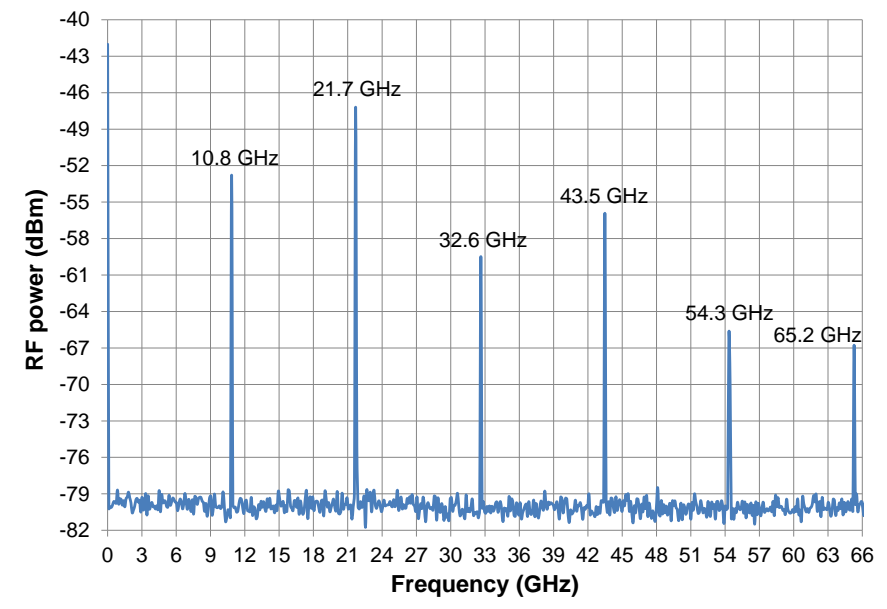

Fig. 10. SBS RF spectrum at the 20m-long FRR's combined outputs, for a $10 \mathrm{dBm}$ laser input power. $\mathrm{Span}=67 \mathrm{GHz} ; \mathrm{RBW}=300 \mathrm{kHz} ; \mathrm{VBW}=20 \mathrm{kHz}$.

\section{4) SBS and Laser RIN Degradation:}

Other than the optical link's NCR degradation resulting from the SBS generation, we have found that the SBS can also have an impact on the laser relative intensity noise (RIN). To estimate this impact, we have measured the laser RIN at the FRR third output while injecting a laser power higher than the first SBST at the resonator input. The results are presented in Fig. 11 and they clearly show the laser RIN degradation between the input and the output of the ring resonator.

We have also measured the Brillouin first Stokes wave RIN at the FRR's second output. We can see that its own low frequency RIN is higher than the laser RIN. This is probably due to the SBS's own Rayleigh-scattered wave, which induces amplitude noise to the SBS wave and degrades its RIN [19]. However, a lower Brillouin RIN than the laser pump has been reported in [27] and [28], but it has been also reported in [29] that Brillouin RIN becomes better than the laser pump's RIN at higher offset frequencies.

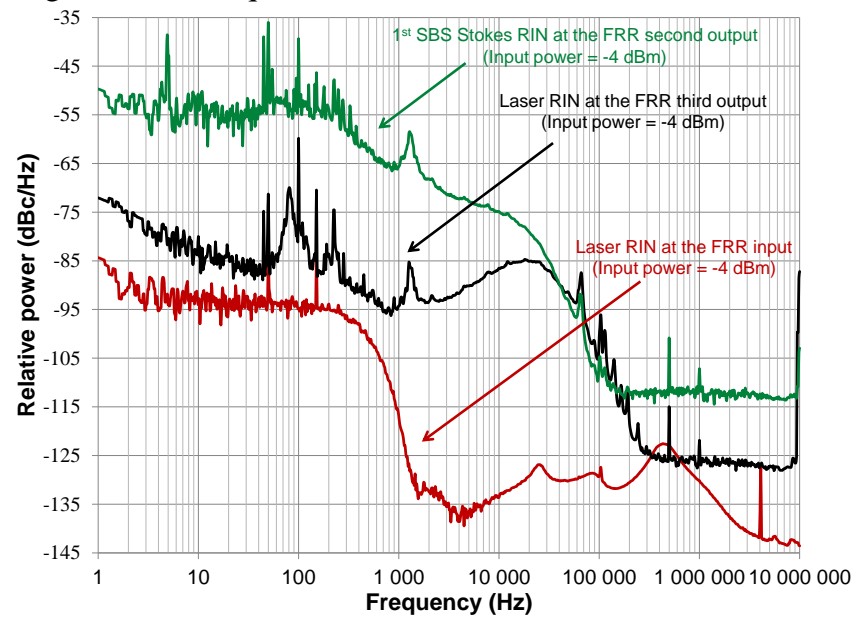

Fig. 11. Laser RIN degradation because of the SBS generation and the first Brillouin Stokes wave RIN measurement.

\section{5) SBS and OEO Phase Noise:}

With all these optical Stokes waves generated around the optical carrier, also adding the weak, frequency up-shifted, anti-Stokes waves (Fig. 4), the carrier depletion, the NCR degradation and the laser RIN degradation, we can expect that our OEO RF signal at $\sim 10 \mathrm{GHz}$ will be severely affected by the presence of the SBS in the FRR. More precisely, it will be affected by the nonlinear noise conversion processes between all these optical carriers. One of these processes is the DC noise detection by the photodiode of all these waves, which is again folded onto the RF carrier either by the photodiode nonlinearity or inside the resonator through interference processes. Therefore, the phase noise of a $10 \mathrm{GHz} \mathrm{RF}$ carrier of the 20m-long FRR based OEO has been carefully measured with an Agilent E5052 B signal source analyzer (SSA) at different laser input power levels. The experimental results are depicted in Fig. 12 and this study is more detailed in [30].

Fig. 12 shows that the $1 / f$ phase noise observed near the carrier is strongly related to the laser power. Indeed, we clearly notice a significant reduction in the close-to-carrier OEO phase noise at lower optical input powers. This phase noise reduction can be about $30 \mathrm{~dB}$ at $10 \mathrm{~Hz}$ offset frequency between two input optical power levels: $2 \mathrm{dBm}$ (above the second SBST) and $-10 \mathrm{dBm}$ (below the first SBST). The increment in the noise floor is directly linked to the NCR which degrades when the carrier power decreases.

These results clearly demonstrate that the optical power at the resonator input must be maintained below the first SBST to get an adequately low phase noise in a FRR based OEO. However, the obtained OEO phase noise shape is still unusual 
and higher than our theoretical expectations when computing the white frequency noise contribution (see the green dashed curve in Fig. 12), and even if the residual phase noise of the microwave amplifiers is included in the model. Some of the possible causes of this phase noise shape and level are the Rayleigh scattering, the spontaneous Brillouin scattering and other laser and photodiode noise conversion phenomenon.

In fact, when operating at low optical power, the SBS is reduced but the photodiode nonlinearity may increase because it has been found that the photodiode we are using in the OEO setup was not working on its optimal input optical power [8]. This may lead to laser's AM and PM noise conversion processes into RF AM and PM noise. Moreover, as it has been reported in [19], the Rayleigh noise spectrum becomes broader at low optical input power, and therefore it can be converted onto the RF signal via the OEO nonlinearity and affect its phase noise at higher offset frequencies.

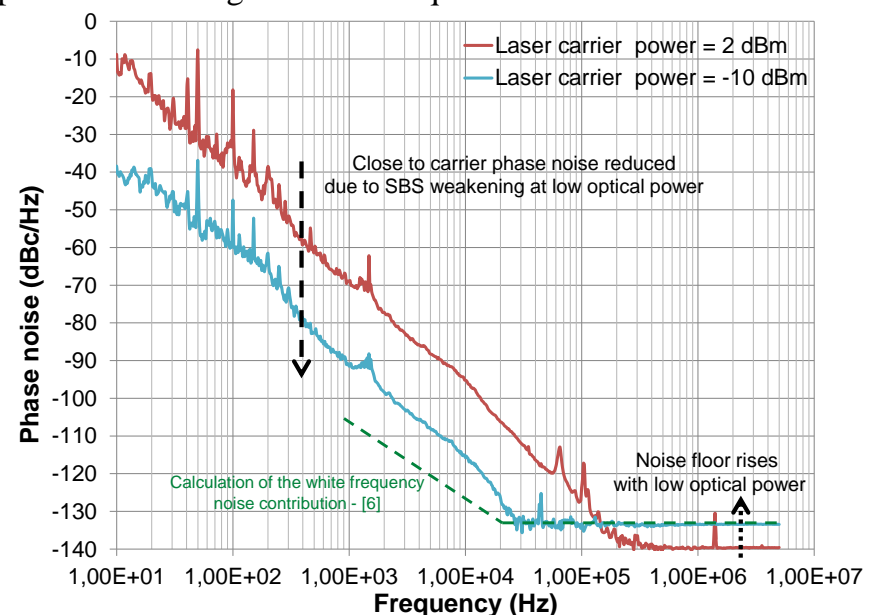

Fig. 12. Phase noise reduction of a 20 meter-long fiber ring resonator based $10 \mathrm{GHz} \mathrm{OEO}$, due to SBS suppression at low optical carrier power. However, the phase noise level remains far from theoretical expectations (dashed curve).

\section{Stimulated Rayleigh Scattering}

\section{1) Stimulated Rayleigh Scattering in Optical Fiber and FRR:}

The Rayleigh scattering occurs from non-propagating density fluctuations (that is why no significant frequency shift follows). This scattering effect is the key element that defines the linear loss $\alpha_{f}$ in optical fibers and the ultimate limitation in the $Q_{O p t}$ factors of optical ring and WGM resonators [31]. The spontaneous Rayleigh scattering is associated to density fluctuations, partly caused by local thermal instabilities in the optical medium [32]. These density fluctuations scatter the laser light wave travelling in the optical fiber or in bulk materials [33]. The electrostriction is the dominant effect that generates stimulated Rayleigh scattering in optical fibers.

The STRS has the potential to degrade the performance of low noise optoelectronic systems [34], such as OEOs. However, it is not the dominant nonlinear optical effect. In fact, the STRS is rapidly buried by the SBS because the SBS gain coefficient is two orders of magnitude higher than that of STRS in long fiber lengths (few km-long optical fibers, [34]).

\section{2) STRS Power Characterization:}

In order to describe the STRS behavior in a FRR, we have first measured its power at the 20m-long FRR second output versus the laser input power (see Fig. 8). We can see that the STRS power increases with the laser input power but it saturates after the Brillouin scattering process starts to be stimulated. Such a Rayleigh-Brillouin scattering behavior has been already reported and thoroughly studied in SPG optical fibers [19] [32]. It has been noticed in [19] that at power levels above SBST, the SBS draws power away from the scattering process, thereby clamping the STRS gain. Moreover, it has been stated that both mechanisms can contribute to the noise induced in RF photonic systems, including delay line OEOs.

\section{3) STRS Spectrum Characterization:}

The STRS gain spectrum can experience anti-Stokes frequency shift due to a coupling between the spontaneous thermal fluctuations and the incident optical light wave. On the other hand, the STRS can experience a Stokes shift due to electrostriction. These frequency shifts are of few $\mathrm{kHz}$ in PM fibers and increase at high input optical power levels [32] [34]. Our FRRs are all made of PM fibers in order to prevent two polarization states behavior of the optical carrier inside the resonator. Therefore, the high intra-cavity power inside the FRR will make the frequency shift of the Rayleigh scattering more detectable.

In order to describe the STRS spectrum behavior in the FRR, and since the STRS frequency shift is very weak, the STRS baseband noise has been measured at the resonator second output. We have used almost the same measurement bench and principle used in [19] and [34] to investigate the STRS spectra in SPG optical fibers. It consists of a crosscorrelation RIN measurement system with the homodyne detection scheme. We used this coherent detection scheme in order to increase the sensitivity of our electrical domain measurements. The measurement bench is depicted in Fig. 13.

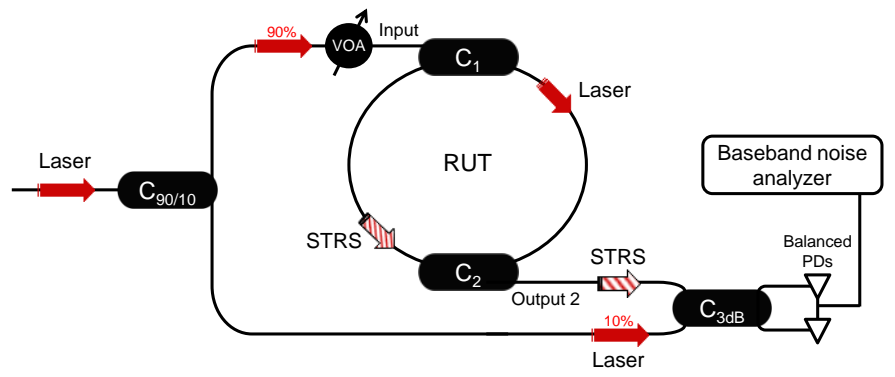

Fig. 13. Backscattered STRS baseband noise measurement bench. VOA: variable optical attenuator; PDs: photodiodes; $\mathrm{C}_{3 \mathrm{~dB}}$ : $3 \mathrm{~dB}$ coupler.

We split off $10 \%$ of the laser carrier power, used as a local oscillator with a constant power, and we send them directly to one of the inputs of a $2 \times 2$ optical coupler. The remaining $90 \%$ of the carrier power is sent to the resonator input. The input level is then controlled using a calibrated variable optical attenuator (VOA). The carrier is then stabilized onto the resonator using the PDH loop. The backscattered SRST gets out naturally from the resonator at its second output and is sent 
to the other input of the $2 \times 2$ optical coupler. The two outputs are then sent to a pair of balanced photodiodes. The output current from the balanced photodiodes is then proportional to the product of the STRS and the laser carrier amplitudes. The baseband noise is then measured and the results are depicted in Fig. 14.

From these results, we can clearly see that the close-tocarrier baseband noise (from $10 \mathrm{~Hz}$ to $200 \mathrm{kHz}$ offset frequencies) increases with the increasing input power. Also, we can see that two peaks appear around $1.5 \mathrm{kHz}$ and $60 \mathrm{kHz}$. Those two peaks testify the presence of the Rayleigh scattering in the noise spectrum and we call them the Rayleigh signature. Moreover, it has been found in [32] that the Rayleigh induced frequency shift is around $1 \mathrm{kHz}$ in PM fibers, and that it increases at high optical power. However, a $50 \mathrm{kHz}$ Rayleigh induced frequency shift has been reported in [34] for single mode fibers. Both studies confirm our reading of the experimental results that we have obtained on the Rayleigh induced noise in the FRR.

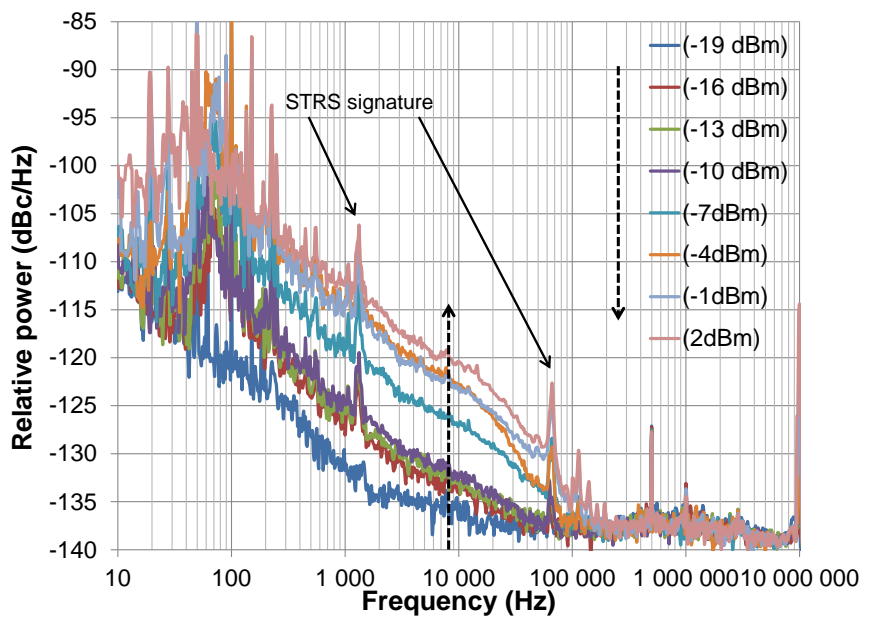

Fig. 14. Baseband noise power spectra at the second output of the 20m-long FRR, measured in the electrical domain using Fig. 13 setup.

\section{4) STRS and Laser RIN Degradation:}

Of course, the laser's Rayleigh-scattered wave is backreflected and naturally gets out from the resonator at its second output. However, as intended for our low noise applications using the FRR in an OEO setup, the most important parameter to scale is the effects of the STRS on the laser carrier directly transmitted through the resonator and emitted from its main output (i.e. the third output of the resonator).

The STRS can effectively contribute to the laser RIN degradation. It has been reported in [35] that a rise in the laser carrier RIN can result from a conversion of the laser phase noise into intensity noise due to a mixing of the laser carrier and the Rayleigh-scattered Stokes wave at the photodiode. We have therefore measured the laser carrier RIN level and behavior at the resonator third output versus the optical input power. The results are depicted in Fig. 15 (for input power levels from $-20 \mathrm{dBm}$ to $-6 \mathrm{dBm}$ ) and in Fig. 16 (for input power levels from $-6 \mathrm{dBm}$ to $7 \mathrm{dBm}$ ).

First of all, to witness the noise induced in the FRR, the
FRR has been replaced by a $3.7 \mathrm{~dB}$ optical attenuator (simulating the same optical loss of the 20m-long FRR). The RIN has been then measured using a very low input power level $(-20 \mathrm{dBm})$ and a classical Koheras laser RIN spectrum has been obtained (see Fig. 15). Now, when the FRR is used, and at the same input power level, the RIN level was higher with a different noise shape and the Rayleigh signature clearly appears. We have measured an almost uniform behavior of the RIN spectrum till below $-6 \mathrm{dBm}$ input power. This noise is generated inside the resonator bandwidth and thus it could affect directly our OEO phase noise. Yet, because of the resonator's high $Q_{o p t}$, this noise is filtered outside of the resonance bandwidth.

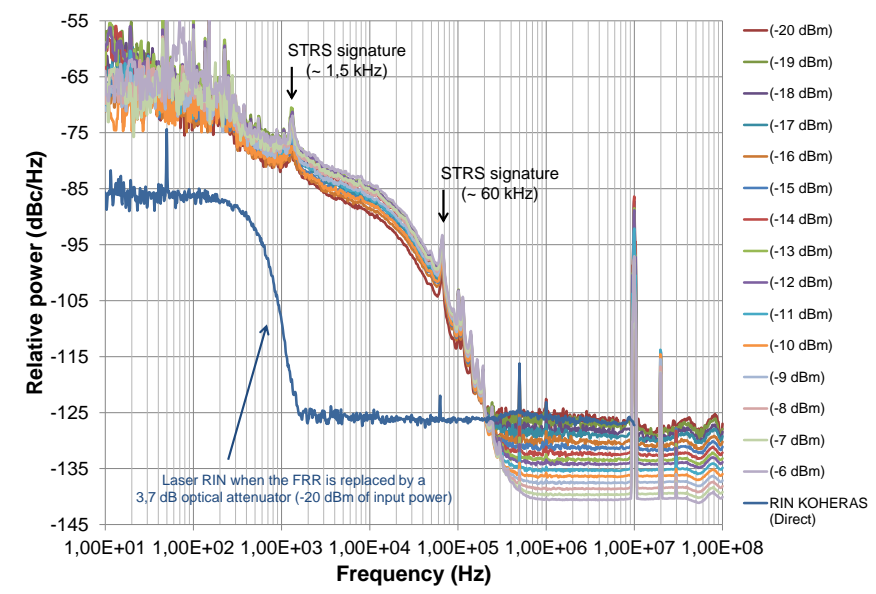

Fig. 15. RIN spectra at the third output of the 20m-long FRR, versus the input optical power (from $-20 \mathrm{dBm}$ to $-6 \mathrm{dBm}$ ), measured directly using one photodiode.

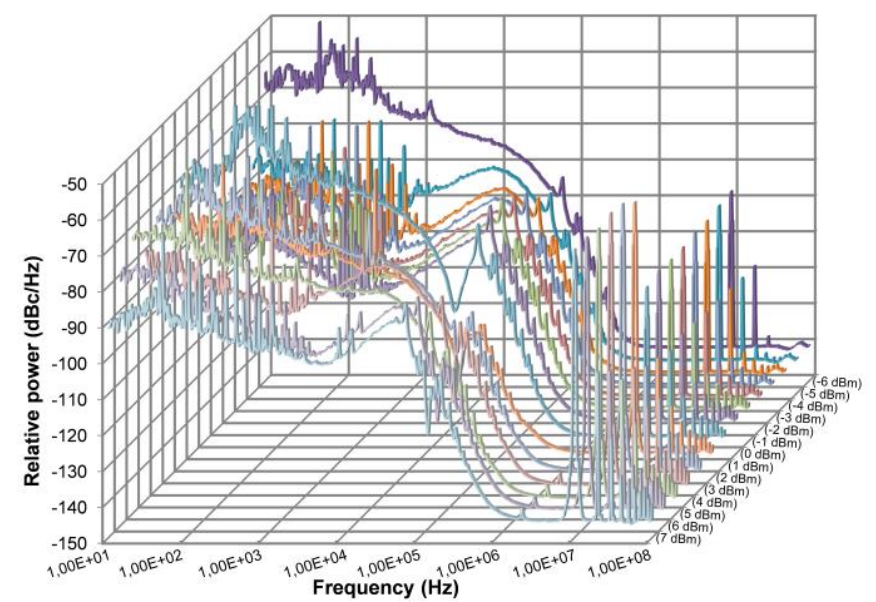

Fig. 16. RIN spectra at the third output of the 20m-long FRR, versus the input optical power (from $-6 \mathrm{dBm}$ to $7 \mathrm{dBm}$ ), measured directly using one photodiode. Frequencies are represented in logarithmic scale.

Now, when the input power starts to be higher than $-6 \mathrm{dBm}$, the RIN level and shape change dramatically (see Fig. 16) but our experimental results were repetitive. Moreover, we can see that the STRS signature is more present around the $60 \mathrm{kHz}$ offset frequency at high optical power levels. The peaks at frequencies multiple of $10 \mathrm{MHz}$ are the 20m-long FRR resonances. This RIN behavior, above $-6 \mathrm{dBm}$ input power, is 
due to the SBS generation inside the resonator. Indeed, the second SBS Stokes wave appears at $-5 \mathrm{dBm}$ input power, as we can see from the SBS power measurement results in Fig. 8, and it gets out from the FRR at its third output. Thus, its baseband noise detected by the photodiode mixes with the baseband noise of the direct transmitted laser wave (already affected by the laser's Rayleigh-scattered wave). To this process we have to add the Rayleigh-scattered waves of the different even SBS Stokes waves. Consequently, the description of the STRS effect on the laser RIN cannot be accurate above $-10 \mathrm{dBm}$ input power (the first SBST). In order to be able to accurately measure only the STRS effect on the laser RIN, it is essential to use a narrow band pass optical filter at the resonator outputs that filters all additional optical components, (eventually SBS Stokes and anti-Stokes waves), around the laser carrier and its own Rayleigh-scattered wave.

\section{Other Nonlinear Optical effects}

\section{1) Four-Wave Mixing in FRR:}

The four-wave mixing (FWM), a third-order nonlinear process, is a type of optical Kerr effect and occurs when light of two or more different wavelengths are launched into an optical fiber with high enough optical power, giving rise to a new wave known as an idler [18]. Generally speaking, the FWM is a kind of optical parametric oscillation and behaves like intermodulation phenomenon in optical systems, similar to the third-order intercept point in electrical systems. The result of the FWM between three different frequencies can lead to the generation of twelve new waves. Three of these new waves overlap when the spacing between the three input frequencies is the same [36].

The FWM is a polarization-dependent process and inversely proportional to the fiber dispersion (more dispersion means less efficiency). It requires phase-matching and high optical power to build up efficiently along the optical fiber. In optical fibers, it can be both harmful and beneficial, depending on the application.

In our FRR, the critical case mentioned above may occur. The generated SBS waves inside the FRR are equally spaced $(10.86 \mathrm{GHz})$ and the phase-matching condition is automatically satisfied. This is because they are all resonant in the FRR, they create each other and they have enough intracavity power inside the resonator, due to the resonant effect (see Fig. 9). The only parameter that may prevent an efficient FWM process in our FRR is the dispersion in the optical fiber we used to fabricate the resonator (D $\sim 17 \mathrm{ps} / \mathrm{nm} . \mathrm{km}$ ). It is probably the reason why we have not observed any FWM in the optical spectrum at the resonator's outputs, even in the presence of the seventh SBS wave, while using the OSA with high amplitude sensitivity ( -60 dBm).

2) Stimulated Raman Scattering in FRR:

The Stimulated Raman scattering (SRS), discovered by C. V. Raman in 1928, is an important nonlinear process that can turn optical fibers into broadband Raman amplifiers and tunable Raman lasers [18]. However, it can severely limit the performance of multichannel optical links by transferring energy from one channel to the neighboring channels.

Fortunately, the SRS threshold (SRST) is very high compared to other nonlinear optical effects. In the FRR case, even with the high power enhancement factor due to the resonance effect, we are far from having SRS generated inside the resonator. If we take the FRR equivalent length approximation mentioned in (2), $\left(L_{\text {eff }}=1180 \mathrm{~m}\right.$ for the $20 \mathrm{~m}$ long FRR), and the Raman threshold definition in [18], we will find that the total intra-cavity power needed to generate the SRS inside the 20m-long FRR will be about $18 \mathrm{~W}$. This means, considering (6), a laser input power of at least $350 \mathrm{~mW}$ $(25.5 \mathrm{dBm})$ at the resonator input. Indeed, SRS has not been observed experimentally in the $20 \mathrm{~m}$-long FRR, even in the presence of the seventh SBS Stokes wave.

\section{OPTIMIZED AND IMMUNIZED FRR AND OEO}

Among all these nonlinear optical effects mentioned in section IV, we have seen that the Brillouin and Rayleigh scatterings can both have the most harmful effects on our FRR characteristics $\left(Q_{o p t}, \mathrm{NCR} \ldots\right)$ and therefore on the FRR OEO phase noise. Of course, by reducing the optical power, we can limit the Brillouin scattering effect and reduce the OEO phase noise [30]; yet, we will still have the spontaneous Brillouin scattering and the Rayleigh scattering effects in addition to the NCR degradation when a low optical power is used. Therefore, high spectral purity signals generation using the FRR based OEO will not be achieved unless we find a solution to suppress all these nonlinear optical effects. It is thus crucial to find a way to mainly suppress the Brillouin scattering and to reduce the Rayleigh scattering, while keeping a high $Q_{O p t}$ and a low transmission loss.

\section{A. SBS Different Suppression Methods}

Many studies on SBS and on the different methods to suppress SBS in optical fibers have been reported in the literature [19], [23], [24], [38] - [41]. The BGS characteristics depends strongly on the temperature (when the temperature increases, the SBS frequency shift and gain increase, and the BGS bandwidth decreases), the strain (when the strain increases, the SBS frequency shift and the BGS bandwidth increase, and the SBS gain decreases), the pressure (when the pressure increases, the SBS frequency shift decrease, but the BGS bandwidth remains constant), the fiber core doping concentration etc..

All these SBS suppression studies were thus concentrated on these parameters: applying temperature or strain distribution to the fiber coil to reduce the SBS [42] - [44]. Still, these methods increase the SBST by only a few decibels. Thus, they are still not efficient, especially for low noise applications and systems such as OEOs.

One other SBS suppression technique is based on spreading the signal spectrum using frequency or phase modulation of the laser carrier. This method can efficiently reduce the SBS 
and STRS in optical fibers by more than $50 \mathrm{~dB}$ [19]. However, spreading the spectrum of the signal may interfere with low phase noise coherent detection schemes similar to those proposed in [22]. Likewise, as we have previously mentioned in section III, the PDH loop we use to stabilize the laser carrier frequency onto the resonator already carries out a phase modulation of the laser carrier for stabilization purpose. Conversely, it seems to have no visible effect on the SBS or on the STRS in the FRR. This is probably because of the discrete resonant frequencies of the resonator. Still, other methods can be used to suppress the SBS while keeping a low noise in optical links. It has been proven in [45] that concatenating multiple fibers, having different BGS frequency shifts each, can lead to an increase in the SBST of about $6 \mathrm{~dB}$; this is however still insufficient for our applications.

\section{B. SBS Suppression Using Optical Isolators}

An elegant method to suppress the SBS in optical fibers, based on the insertion of optical isolators positioned at alternative distances within a SPG optical link, may also be implemented within a FRR for the same purpose [46]. The isolation of the different isolators will thus prevent the SBS from intensifying in the backward direction in the optical fiber.

Essentially, in SPG optical fibers, the generated SBS Stokes wave travels backwards and it is amplified by the Brillouin gain as it has been already explained in section IV-B-1. Now, when an optical isolator is inserted in a fiber with a total length $\left(\mathrm{L}_{\mathrm{T}}\right)$, having its own effective length $L_{\text {eff }}$ and SBST, the isolator virtually cuts the fiber into two new lengths $\left(L_{1}\right.$ and $\left.L_{2}\right)$ regarding the Brillouin gain. Consequently, the SBS interaction between the fiber's two sections is absent and the fiber SBST is increased because it is replaced by two other SBSTs related to the new fiber sections, $\mathrm{L}_{1}$ and $\mathrm{L}_{2}$. The SBS Stokes wave will be therefore completely cut off by the isolator in the fiber link. However, to prevent the SBS from being generated in long optical links, the length of each fiber section (the sections are separated by isolators) must respect the launched power into each fiber section input in order to not exceed the section SBST.

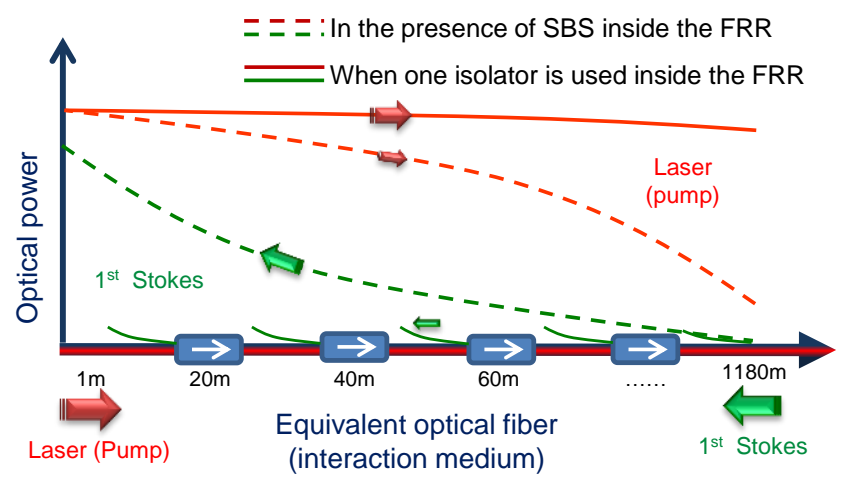

Fig. 17. Illustration of the laser (pump) and the first SBS behavior inside the 20m-long FRR, with and without one isolator inserted inside the FRR

In the FRR case, this method is of great interest because it is much more efficient in a FRR geometry than in a SPG optical fiber. Indeed, if one ideal isolator (with zero insertion loss and infinite isolation) is inserted inside the FRR, it will completely prevent the SBS from building-up inside the resonator. This is due to the fact that, virtually, we will have a number $(N)$ of isolators equal to the photon's total number of laps around the resonator $\left(N=L_{e q} / L\right)$. These virtual isolators will be spaced by the FRR physical length $L$. Therefore, the SBS Stokes wave will be completely cut off by these virtual isolators, and the SBST inside the resonator will be increased to be the one of the physical resonator length $L$, not the SBST of the FRR's equivalent length $L_{e q}$, (see Fig. 17 for the method illustration if applied to the 20m-long FRR case).

For example, the SBST of the 20m-long FRR will be increased from $-1.4 \mathrm{dBm}$ (for a $\sim 1180 \mathrm{~m}$ virtual length) to $33.5 \mathrm{dBm}$ (for a $20 \mathrm{~m}$ physical length), thereupon by approximately $35 \mathrm{~dB}$. The presence of the isolator in the FRR will also efficiently reduce the Rayleigh scattering intensity.

\section{Optimized and Immunized FRR}

Unfortunately, and despite the fact that the SBS will be suppressed and the STRS will be reduced, the optical isolator is not ideal, as it does not provide zero insertion loss and infinite isolation. Thus, in the FRR case, the isolator insertion loss will be also multiplied by the photons total laps $N$. Therefore, the FRR $Q_{o p t}$ will be reduced unless we optimize the resonator's different parameters (length, coupling factor, architecture, loss...) to compensate the isolator loss and get a SBS suppression, a STRS reduction, a satisfying quality factor, a low transmission loss and finally a good phase noise result once the device is included in an OEO setup. This optimization study has been performed on a FRR model that we have developed using a microwave simulation software, ADS (Agilent). The modeling of the resonator using ADS is detailed in [47].

In Fig. 18 and Fig. 19, we present quality factor, intra-cavity power and transmission loss ADS simulation results obtained for a $100 \mathrm{~m}$-long FRR. We have found that an optimized coupling factor of $8 \%$, while mainly taking the resonator's transmission loss into account, must lead to a $92 \mathrm{kHz}$ FWHM, and therefore to a $Q_{O p t}$ of $2.1 \times 10^{9}\left(Q_{R F}\right.$ of $1.1 \times 10^{5}$ at $f_{R F}=10 \mathrm{GHz}$ ), with a $2 \mathrm{MHz} F S R$, a $4 \mathrm{~dB}$ optical transmission loss, a $7 \mathrm{~dB}$ power enhancement factor and a $22.7 \mathrm{~dB}$ out-of-band rejection.

A $Q_{O p t}$ of $2.1 \times 10^{9}$ means an equivalent length $L_{e q}$ of $708 \mathrm{~m}$, and consequently an intra-cavity SBST of $64 \mathrm{~mW}(18 \mathrm{dBm})$, according to the aforementioned theoretical approach. Furthermore, with a $7 \mathrm{~dB}$ intra-cavity power enhancement factor, the resonator input power SBST will be about $12.7 \mathrm{~mW}$ $(11 \mathrm{dBm})$, always according to the SBST definition in [18]. Therefore, if the SBS is generated inside the resonator, it will be detected far below this SBST value (at least $5 \mathrm{~dB}$ below this SBST theoretical value and definition, see the $20 \mathrm{~m}$-long FRR SBST power measurements in Fig. 8).

In the new 100m-long PM FRR structure, we have added an inline polarizer at the resonator input. This will completely eliminate the possibility of two polarization states behavior of the optical carrier at the FRR input. Essentially, the PM fiber 
used in the resonator only maintains these states of polarization and do not ensure only one polarization state behavior. The 100m-long immunized FRR (IFRR) architecture is depicted in Fig. 20.

Once the 100m-long IFRR has been fabricated, all the experimental results obtained had met our expectations. First, the IFRR has been characterized optically using the same setup of Fig. 7 and the results are depicted in Fig. 21.

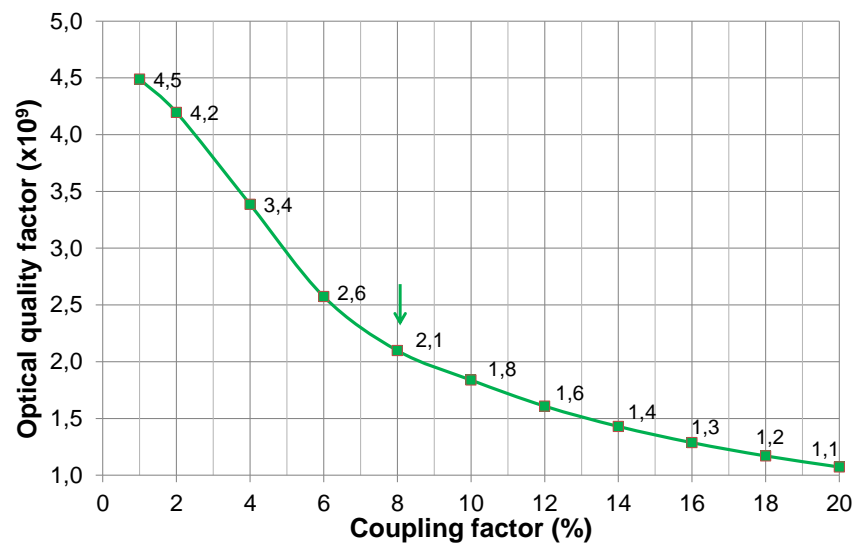

Fig. 18. 100m-long FRR optical quality factor versus the coupling factor (for both couplers), simulated using FRR ADS model.

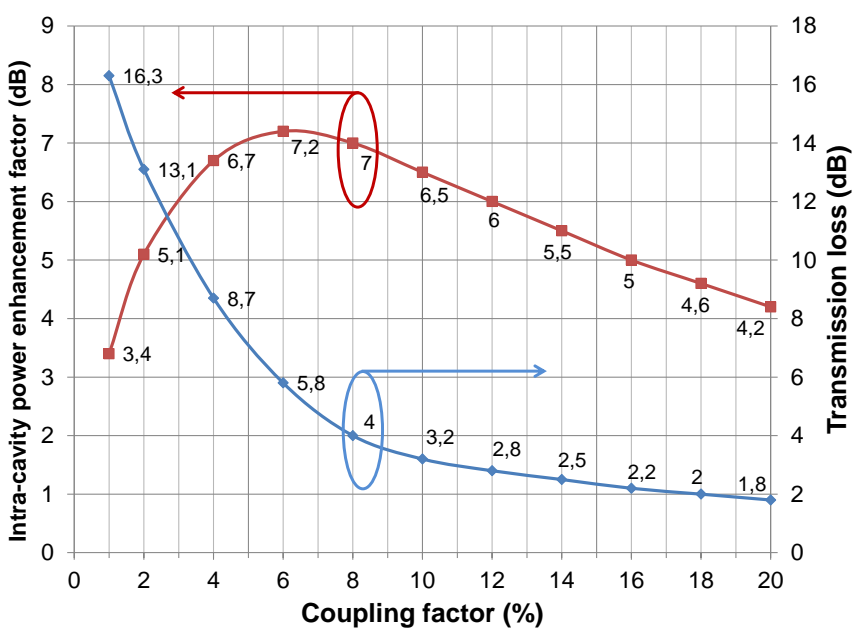

Fig. 19. 100m-long FRR intra-cavity power enhancement factor and transmission loss versus the coupling factor (for both couplers), simulated using FRR ADS model.

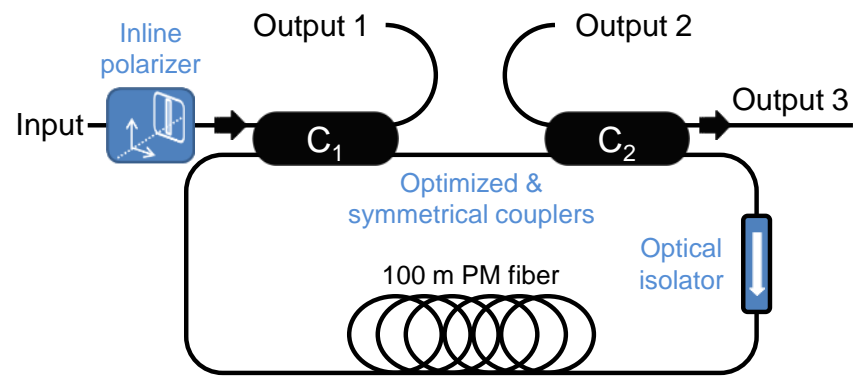

Fig. 20. New 100m-long immunized FRR architecture.

We can clearly see from these results that the SBS has been completely suppressed. No carrier saturation has been observed (the carrier power at the resonator output increases linearly with a slope equal to 1 ; about $4 \mathrm{~dB}$ optical loss and
$7 \mathrm{~dB}$ power enhancement factor have been obtained) and the STRS intensity has been efficiently reduced (more than $40 \mathrm{~dB}$ rejection). The optical $4 \mathrm{~dB}$ power loss and $7 \mathrm{~dB}$ power enhancement factor have been also accurately measured using an optical power meter.

The IFRR characterization results in the RF domain also met our expectations: $Q_{O p t}$, out-of-band rejection, FSR, RF transmission loss (doubled in the RF domain because of the photodiode quadratic detection); see Fig. 22. These characteristics were accurately measured using a high resolution vector network analyzer [15].

All these experimental results prove that we have now an optimized resonator with a high $Q_{O p t}$ and low transmission loss. Also, the resonator is completely immunized against the SBS, and the STRS is efficiently reduced. The use of such resonator as the frequency stability element in an OEO setup should now considerably reduce its phase noise.

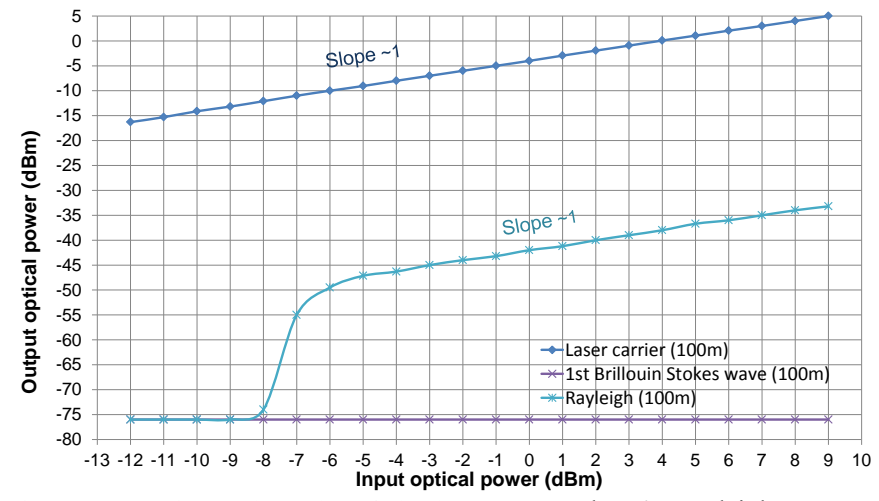

Fig. 21. Experimental results of the laser output, laser's Rayleigh-scattered wave and first SBS Stokes wave power measurements in the $100 \mathrm{~m}$-long IFRR, versus the laser input optical power.

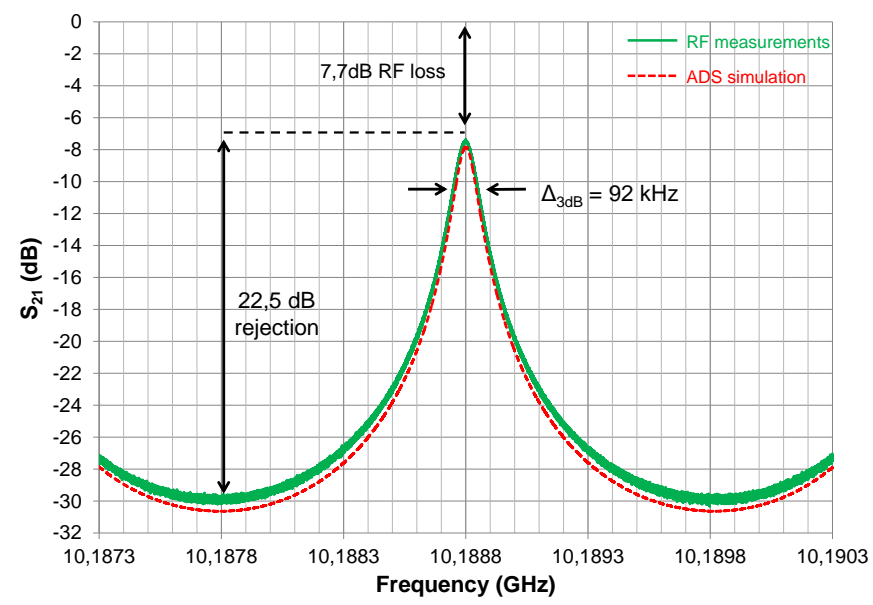

Fig. 22. RF transmission characteristics of the $100 \mathrm{~m}$-long IFRR

\section{OEO Based on an Optimized and Immunized FRR}

When the new $100 \mathrm{~m}$-long IFRR is used in an OEO setup, the OEO noise modeling, detailed in [6], expects a phase noise level of $-134 \mathrm{dBc} / \mathrm{Hz}$ at $10 \mathrm{kHz}$ offset frequency (see the dashed curve in Fig. 23). But if we add the phase noise contribution of the optical and the RF amplifiers we use in our experimental OEO setup, we can expect a $-128 \mathrm{dBc} / \mathrm{Hz}$ phase 
noise level at $10 \mathrm{kHz}$ offset frequency (see the blue curve in Fig. 23).

Finally, the $100 \mathrm{~m}$ IFRR resonator has been included in the OEO experimental setup and a considerably reduced OEO phase noise has been measured, with a $-128 \mathrm{dBc} / \mathrm{Hz}$ noise level at $10 \mathrm{kHz}$ offset frequency and a $-150 \mathrm{dBc} / \mathrm{Hz}$ noise floor as theoretically predicted (see the green curve in Fig. 23). The rapid increase in the close-to-carrier OEO phase noise is likely due to the thermal effects because our OEO setup is a laboratory system and all the OEO parts were exposed in free space and not thermally isolated, including the resonator.

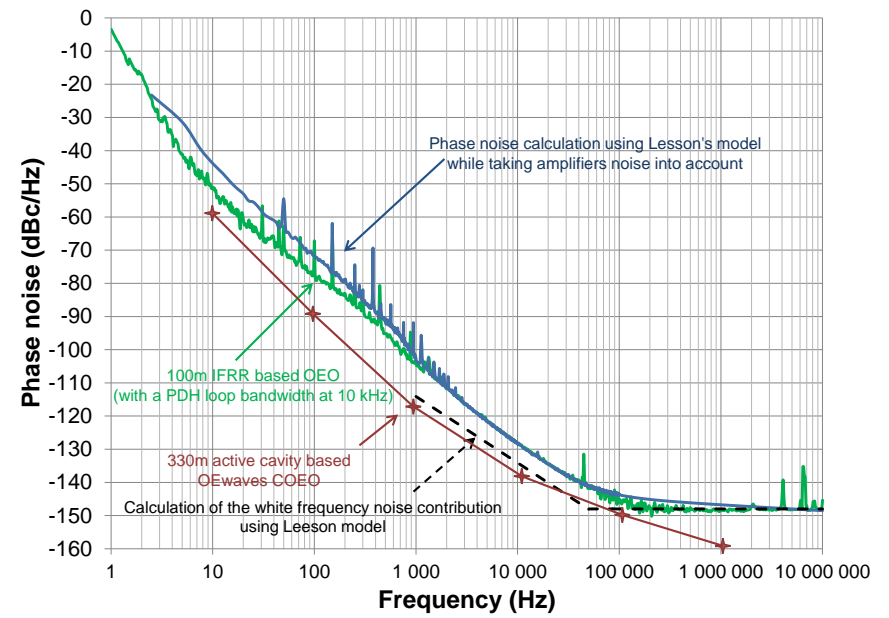

Fig. 23. 100m-long passive IFRR based OEO: phase noise measurement, calculation (based on measured amplifiers' residual phase noise) and comparison with OEwaves' $330 \mathrm{~m}$ active-cavity based COEO

The results obtained with the $100 \mathrm{~m}$-long IFRR OEO are very encouraging if compared with the $330 \mathrm{~m}$ active-cavity based OEO, also known as coupled optoelectronic oscillator (COEO) and currently commercialized by OEwaves [48], (see the red-stars curve in Fig. 23). Moreover, we can expect to get an even better result than the one presented in Fig. 23, if a lower loss isolator is used, if RF amplifiers' noise contribution could be reduced (the OEO phase noise is clearly limited by amplifiers noise), and if other low noise components are used (particularly the laser). Indeed, a $7 \mathrm{~dB}$ reduction in the OEO phase noise at $10 \mathrm{kHz}$ offset frequency could be theoretically obtained if the RF amplifiers noise contribution is reduced.

\section{CONCLUSION}

Theoretical and experimental studies were performed to reduce the phase noise of a passive fiber ring resonator (FRR) based optoelectronic oscillator (OEO). It has been found that the optical scattering induced noise inside the resonator can severely affect the oscillator stability, especially the Brillouin and Rayleigh scatterings. These nonlinear optical effects were extensively studied in order to understand their behavior inside the FRR, and then to optimize the resonator architecture in order to suppress them. Our optimization studies have led to the design of a new optimized and immunized resonator with high optical quality factor. Phase noise levels of $-50 \mathrm{dBc} / \mathrm{Hz}$ and $-128 \mathrm{dBc} / \mathrm{Hz}$ at respectively $10 \mathrm{~Hz}$ and $10 \mathrm{kHz}$ offset frequencies have been measured in a $100 \mathrm{~m}$-long passive-FRR based OEO. Improvement is still possible if lower loss components are used in the resonator architecture, and if lower noise amplifiers and components are used in the OEO setup.

\section{REFERENCES}

[1]. G. Cibiel, M. Regis, O. Llopis, A. Rennane, L. Bary, R. Plana, Y. Kersale, and V. Giordano, "Optimization of an ultra-low phase noise sapphire-SiGe HBT oscillator using nonlinear CAD," Ultrasonics, Ferroelectrics and Frequency Control, IEEE Transactions on , vol.51, no.1, pp.33-41, Jan. 2004.

[2]. X. S. Yao and L. Maleki, "Optoelectronic microwave oscillator," J. Opt. Soc. Am. B 13, 1725-1735, 1996.

[3]. X. S. Yao, and L. Maleki, "Progress in the optoelectronic oscillator - a ten year anniversary review," IEEE Microwave Theory and Tech. Symp. Digest, 2004, pp. 287-290.

[4]. O. Okusaga, E. J. Adles, E. C. Levy, W. Zhou, G. M. Carter, C. R. Menyuk, and M. Horowitz, "Spurious mode reduction in dual injection-locked optoelectronic oscillators," Opt. Express 19, 58395854, 2011.

[5]. A. A. Savchenkov, V. S. Ilchenko, A. B. Matsko and L. Maleki, "Kilohertz optical resonances in dielectric crystal cavities," Phys. Rev. A 70, 05804, 2004.

[6]. A. Bouchier, K. Saleh, P. H. Merrer, O. Llopis and G. Cibiel, "Theoretical and experimental study of the phase noise of optoelectronic oscillators based on high quality factor optical resonators," Proc. of the 2010 IEEE-IFCS, pp. 544-548.

[7]. K. Saleh, A. Bouchier, P. H. Merrer, O. Llopis and G. Cibiel, "Fiber ring resonator based opto-electronic oscillator: phase noise optimisation and thermal stability study," Proc. SPIE 7936, 79360A, 2011.

[8]. K. Saleh, P.H. Merrer, O. Llopis, G. Cibiel, "Optoelectronic oscillator based on fiber ring resonator: Overall system optimization and phase noise reduction," Frequency Control Symposium (FCS), 2012 IEEE International, vol., no., pp.1-6, 21-24.

[9]. L. F. Stokes, M. Chodorow, and H. J. Shaw, "All-single-mode fiber resonator," Opt. Lett. 7, pp. 288-290, 1982.

[10]. S. Norcia, S. Tonda-Goldstein, D. Dolfi, J. Huignard, and R. Frey, "Efficient single-mode Brillouin fiber laser for low-noise optical carrier reduction of microwave signals," Opt. Lett. 28, pp. 1888-1890, 2003.

[11]. T. Zhu, X. Bao, and L. Chen, "A Single Longitudinal-Mode Tunable Fiber Ring Laser Based on Stimulated Rayleigh Scattering in a Nonuniform Optical Fiber," J. Lightwave Technol. 29, pp. 1802-1807 (2011).

[12]. K. Saleh, P. H. Merrer, O. Llopis, and G. Cibiel, "Millimeter Wave Generation Using Brillouin Scattering in a High Q Fiber Ring Resonator," Proc. of the 2012 IEEE International Topical Meeting on Microwave Photonics (MWP), Noordwjik, sept. 2012.

[13]. X. S. Yao, "High-quality microwave signal generation by use of Brillouin scattering in optical fibers," Opt. Lett. 22, 1329-1331, 1997.

[14]. P.H. Merrer, H. Brahimi, O. Llopis, "Optical techniques for microwave frequency stabilization : Resonant versus delay line approaches and related modelling problems," Microwave photonics, 2008. jointly held with the 2008 asia-pacific microwave photonics conference. mwp/apmp 2008. international topical meeting on , vol., no., pp.146149, Sept. 9 2008-Oct. 32008.

[15]. P. H. Merrer, A. Bouchier, H. Brahimi, O. Llopis, and G. Cibiel, "HighQ Optical Resonators for Stabilization of High Spectral Purity Microwave Oscillators," proc. of the 2009 IEEE EFTF-IFCS, pp. 866869.

[16]. F. Zhang and J. Lit, "Direct-coupling single-mode fiber ring resonator," J. Opt. Soc. Am. A 5, pp. 1347-1355, 1988.

[17]. R. W. P. Drever, J. L. Hall, F. V. Kowalski, J. Hough, G. M. Ford, A. J. Munley, and H. Ward, "Laser phase and frequency stabilization using an optical resonator," Appl. Phys. B, vol. 31, pp. 97-105, 1983.

[18]. G.P.Agrawal, "Nonlinear Fiber Optics," 4th ed. (Academic Press 2007), chapters $(8,9,10)$. 
[19]. O. Okusaga, J. Cahill, W. Zhou, A. Docherty, G. M. Carter, C. R. Menyuk, "Optical scattering induced noise in RF-photonic systems," Frequency Control and the European Frequency and Time Forum (FCS), 2011 Joint Conference of the IEEE International, vol., no., pp.1-6.

[20]. A.I. Filipenko, "End face nonperpendicularity analysis in fiber connectors," Laser and Fiber-Optical Networks Modeling, 2001. Proceedings of LFNM 2001. 3rd International Workshop on, vol., no., pp. 53-55.

[21]. L. Brillouin, "Diffusion de la lumiere par un corps transparents homogene," Ann. Phys. 17, $88,1922$.

[22]. A. Kobyakov, M. Sauer, and D. Chowdhury, "Stimulated Brillouin Scattering in Optical Fibers," Advances in Optics and Photonics, vol. 2, pp. 1-59, 2010 .

[23]. Q. Yu, X. Bao, and L. Chen, "Temperature dependence of Brillouin frequency, power, and bandwidth in panda, bow-tie, and tiger polarization-maintaining fibers," Opt. Lett. 29, pp. 17-19, 2004.

[24]. M. Nikles, L. Thevenaz, and P.A. Robert, "Brillouin gain spectrum characterization in single-mode optical fibers," Lightwave Technology, Journal of, vol.15, no.10, pp. 1842-1851, Oct 1997.

[25]. X.S. Yao, "Brillouin selective sideband amplification of microwave photonic signals," Photonics Technology Letters, IEEE , vol.10, no.1, pp.138-140, Jan. 1998.

[26]. A. Debut, S. Randoux, and J. Zemmouri, "Linewidth narrowing in Brillouin lasers: theoretical analysis," Phys. Rev. A 62(2), 023803, 2000.

[27]. J. Geng; S. Staines, Z. Wang, J. Zong, M. Blake, and S. Jiang, "Highly stable low-noise Brillouin fiber laser with ultranarrow spectral linewidth," Photonics Technology Letters, IEEE , vol.18, no.17, pp.1813-1815, Sept. 2006

[28]. L. Stépien, S. Randoux, and J. Zemmouri, "Intensity noise in Brillouin fiber ring lasers," J. Opt. Soc. Am. B 19, pp. 1055-1066, 2002.

[29]. Y. Song and X. Zhang, "High power low RIN Brillouin fiber laser with optoelectronic oscillator configuration", Proc. SPIE 6838, 68380T (2007).

[30]. K. Saleh, P. H. Merrer, O. Llopis, and G. Cibiel, "Optical scattering noise in high Q fiber ring resonators and its effect on optoelectronic oscillator phase noise," Opt. Lett. 37, pp. 518-520, 2012.

[31]. M. Borselli, K. Srinivasan, P. E. Barclay, and O. Painter, "Rayleigh scattering, mode coupling, and optical loss in silicon microdisks," Appl. Phys. Lett. 85, 3693, 2004.

[32]. T. Zhu, X. Bao, L. Chen, H. Liang, and Y. Dong, "Experimental study on stimulated Rayleigh scattering in optical fibers," Opt. Express 18, 22958-22963, 2010.

[33]. R. W. Boyd, "Nonlinear Optics," 3rd ed., (Academic Press, 2008).

[34]. O. Okusaga, J. Cahill, A. Docherty, W. Zhou, and C. Menyuk, "Guided entropy mode Rayleigh scattering in optical fibers," Opt. Lett. 37, pp. 683-685, 2012.

[35]. A.B. Ruffin, "Stimulated brillouin scattering: an overview of measurements, system impairments, and applications," Optical Fiber Measurements, 2004. Technical Digest: Symposium on , vol., no., pp. 23- 28.

[36]. N. Shibata, R. Braun, and R. Waarts, "Phase-mismatch dependence of efficiency of wave generation through four-wave mixing in a singlemode optical fiber," Quantum Electronics, IEEE Journal of , vol.23, no.7, pp. 1205- 1210, 1987.

[37]. C.M. McIntosh, A.G. Grandpierre, D.N. Christodoulides, J. Toulouse, and J.-M.P. Delavaux, "Eliminating SRS channel depletion in massive WDM systems via optical filtering techniques," Photonics Technology Letters, IEEE, vol.13, no.4, pp.302-304, Apr. 2001.

[38]. Q. Yu, X. Bao, and L. Chen, "Strain dependence of Brillouin frequency, intensity, and bandwidthin polarization-maintaining fibers," Opt. Lett. 29, pp. 1605-1607, 2004.

[39]. T. Parker, M. Farhadiroushan, V. Handerek, and A. Rogers, "Temperature and strain dependence of the power level and frequency of spontaneous Brillouin scattering in optical fibers," Opt. Lett. 22, 787-789, 1997.

[40]. W. Zou, Z. He, and K. Hotate, "Complete discrimination of strain and temperature using Brillouin frequency shift and birefringence in a polarization-maintaining fiber," Opt. Express 17, 1248-1255, 2009.
[41]. S. L. Floch and P. Cambon, "Study of Brillouin gain spectrum in standard single-mode optical fiber at low temperatures $(1.4-370 \mathrm{~K})$ and high hydrostatic pressures (1-250 bars)," Opt. Commun. 219, pp. 395410, 2003.

[42]. J. Hansryd, F. Dross, M. Westlund, P.A. Andrekson, and S.N. Knudsen, "Increase of the SBS threshold in a short highly nonlinear fiber by applying a temperature distribution," Lightwave Technology, Journal of , vol.19, no.11, pp. 1691-1697, Nov. 2001.

[43]. N. Yoshizawa, T. Horiguchi, T. Kurashima, "Proposal for stimulated Brillouin scattering suppression by fibre cabling," Electronics Letters, vol.27, no.12, pp. 1100-1101, June 1991.

[44]. R. Engelbrecht, M. Mueller, and B. Schmauss, "SBS shaping and suppression by arbitrary strain distributions realized by a fiber coiling machine" IEEE/LEOS Winter Topicals Meeting Series, 2009 , vol., no., pp. 248-249.

[45]. J. Cahill, O. Okusaga, and J. White, "Suppression of Stimulated Brillouin Scattering in High Power, Low phase noise RF-Photonic links," Frequency Control Symposium (FCS), 2012 IEEE International , vol., no., pp. 1-4.

[46]. Y. Takushima, and T. Okoshi, "Suppression of stimulated Brillouin scattering using optical isolators," Electronics Letters, vol.28, no.12, pp. 1155-1157, June 1992.

[47]. K. Saleh, P.H. Merrer, A. Ali-Slimane, O. Llopis, and G. Cibiel, "Study of the Noise Processes in Microwave Oscillators Based on Passive Optical Resonators," Accepted for publication in the special issue of the International Journal of Microwave and Wireless Technologies.

[48]. D. Eliyahu, and L. Maleki, "Modulation response (S21) of the coupled opto-electronic oscillator," Frequency Control Symposium and Exposition, 2005. Proceedings of the 2005 IEEE International, vol., no., pp. 850-856.

Khaldoun Saleh received his M.S. degree in 2009 and his Ph.D. degree in 2012, both in microwaves, electromagnetism and optoelectronics from Toulouse III University, France. He is currently working at the Laboratory of Analysis and Architecture of Systems (LAAS-CNRS) and the French National Space Centre (CNES), Toulouse, France. He is working on the improvement of high spectral purity microwave sources based on optical resonators. He investigates the different noise conversion phenomena in different optoelectronic oscillators based either on fiber ring resonators or on whispering gallery mode micro-resonators. His studies are particularly focused on the optical scattering induced noise in these oscillators and the beneficial use of the nonlinear optical effects generated in optical resonators.

Olivier Llopis received the engineer diploma from ENSTB, Brest, in 1987 and the Ph.D. degree in Electronics from the University Paul Sabatier, Toulouse, in 1991. He is currently working with the French National Centre for Scientific Research (C.N.R.S), in the Laboratoire d'Analyse et d'Architecture des Systèmes (L.A.A.S) in Toulouse. He is currently leading the team "Microwave and Opto-microwave Systems for Telecommunications". His personal research interests are in the study of microwave sources, both with theoretical and experimental approaches, and on the interaction of microwave and optics. He has proposed different techniques to investigate the phase noise in microwave oscillators. He is today deeply involved in the development of optical-microwave systems for time \& frequency applications. He has proposed specific modeling techniques for microwave-optical systems and has developed new approaches for microwave generation using optics.

Gilles Cibiel was born in Castres, France, on January 26, 1976. He received the Ph.D. degree in optic and microwave communications at the Laboratory of Analysis and Architecture (LAAS) of Systems of the French National Center for Scientific Research (CNRS), Toulouse, France. Since 2004, he joined the Microwave and Time-Frequency Deptartement of the French spatial agency (Centre National d'Etudes Spatiales). His current research interests are sources dedicated to space applications including oscillators based on quartz, MEMS, sapphire and optical microresonator, and, the development of specific phase noise measurement techniques at radio and microwave -frequency. 